\title{
An experimental and theoretical study of the valence shell photoelectron spectra of 2-chloropyridine and 3-chloropyridine
}

D. M. P. Holland, ${ }^{1, a)}$ I. Powis ${ }^{2}$ A. B. Trofimov, ${ }^{3,4}$ R. C. Menzies, ${ }^{2}$ A. W. Potts,${ }^{5}$ L. Karlsson, ${ }^{6}$ I. L. Badsyuk, ${ }^{3}$ T. E. Moskovskaya, ${ }^{3}$ E. V. Gromov, ${ }^{7}$ and J. Schirmer ${ }^{7}$

${ }^{1}$ Daresbury Laboratory, Daresbury, Warrington, Cheshire WA4 4AD, UK

${ }^{2}$ School of Chemistry, University of Nottingham, Nottingham, NG7 2RD, UK

${ }^{3}$ Laboratory of Quantum Chemistry, Irkutsk State University, Karl Marx Str. 1, 664003 Irkutsk, Russia

${ }^{4}$ Favorsky's Institute of Chemistry, SB RAS, Favorsky Str. 1, 664033 Irkutsk, Russia

${ }^{5}$ Department of Physics, King's College, Strand, London WC2R 2LS, UK

${ }^{6}$ Department of Physics and Astronomy, Uppsala University, Box 516, SE-75120 Uppsala,

\section{Sweden}

${ }^{7}$ Theoretische Chemie, Physikalisch-Chemisches Institut, Universität Heidelberg, Im Neuenheimer Feld 229, 69120 Heidelberg, Germany

\section{Abstract}

The valence shell photoelectron spectra of 2-chloropyridine and 3-chloropyridine have been studied both experimentally and theoretically. Synchrotron radiation has been employed to record angle resolved photoelectron spectra in the photon energy range $20-100 \mathrm{eV}$, and these have enabled anisotropy parameters and branching ratios to be derived. The experimental results have been compared to theoretical predictions obtained using the continuum multiple scattering $\mathrm{X} \alpha$ approach. This comparison shows that the anisotropy parameter associated with the nominally chlorine lone-pair orbital lying in the molecular plane is strongly affected by the atomic Cooper minimum. In contrast, the photoionization dynamics of the second lone-pair orbital, orientated perpendicular to the molecular plane, seem relatively unaffected by this atomic phenomenon. The outer valence ionization has been studied theoretically using the third-order algebraic-diagrammatic construction approximation scheme $(\mathrm{ADC}(3))$ for the one-particle Green's function, the outer valence Green's function method (OVGF) and the equation-of-motion (EOM) coupled cluster (CC) theory at the level of the EOM-IP-CCSD and EOM-EE-CC3 models. The convergence of the results to the complete basis set limit has been investigated. The ADC(3) method has been employed to compute the complete valence shell ionization spectra of 2-chloropyridine and

a)Electronic address: david.holland@stfc.ac.uk 
Publishing 3-chloropyridine. The relaxation mechanism for ionization of the nitrogen $\sigma$-type lone-pair orbital ( $\left.\sigma_{N} L P\right)$ has been found to be different to that for the corresponding chlorine lone-pair $\left(\sigma_{\mathrm{Cl} L P}\right)$. For the $\sigma_{\mathrm{N}} \mathrm{LP}$ orbital, $\pi-\pi^{*}$ excitations play the main role in the screening of the lonepair hole. In contrast, excitations localized at the chlorine site involving the chlorine $\pi \mathrm{Cl} \mathrm{LP}$ lone-pair and the $\mathrm{Cl} 4 \mathrm{p}$ Rydberg orbital are the most important for the $\sigma_{\mathrm{Cl}} \mathrm{LP}$ orbital. The calculated photoelectron spectra have allowed assignments to be proposed for most of the structure observed in the experimental spectra. The theoretical work also highlights the formation of satellite states, due to the breakdown of the single particle model of ionization, in the inner valence region. 


\section{Publishing I. INTRODUCTION}

Pyridine $\left(\mathrm{C}_{5} \mathrm{H}_{5} \mathrm{~N}\right.$, Figure 1) is the simplest six-membered nitrogen-containing aromatic molecule which appears as a structural unit in many biological and biologically active compounds. The basic pyridine derivatives, particularly the monohalogenated pyridines, are useful models for investigating substitution effects in the six-membered heteroaromatic ring. Such studies improve our understanding of the chemical reactivity of the ubiquitous nitrogencontaining organic compounds and also provide information on their physical properties (e.g., orbital structure and photoionization dynamics).

The outer valence shell electronic structure of pyridine has been the subject of extensive experimental and theoretical investigations (Ref. 1, and references therein). The nature of the electron distribution is rather complex, and only high-level theoretical methods appear to be appropriate. Specifically, the molecular orbital (MO) sequence, omitting the Kshell orbitals, which is predicted to be $\ldots 5 \mathrm{~b}_{2}(\sigma) 7 \mathrm{a}_{1}\left(\sigma_{\mathrm{N} L P}\right) 2 \mathrm{~b}_{1}(\pi) 1 \mathrm{a}_{2}(\pi)$ by Hartree-Fock (HF) calculations, is modified to ... $5 \mathrm{~b}_{2}(\sigma) 2 \mathrm{~b}_{1}(\pi) 1 \mathrm{a}_{2}(\pi) 7 \mathrm{a}_{1}\left(\sigma_{\mathrm{N} L P}\right)$ when more sophisticated approaches are employed. ${ }^{1}$ The 7a1 orbital formally represents the non-bonding $\sigma$-type nitrogen lone-pair orbital, and its change in position in the molecular orbital sequence is a consequence of strong orbital relaxation and electron correlation effects which accompany ionization of this orbital. The contributions from these two effects have been discussed in detail by Trofimov et al, ${ }^{1}$ where the results from various theoretical methods are reported. In fact, high-level electronic structure calculations lead to ionization energies of the $7 \mathrm{a}_{1}$ and $1 \mathrm{a}_{2}$ orbitals which are very similar to each other. This prediction is consistent with the experimental photoelectron spectra which show that the bands due to the two outermost orbitals strongly overlap..$^{2-4}$

In order to improve our understanding of the differing extent to which orbital relaxation and electron correlation affect orbitals of alternative type $(\sigma, \pi$, or non-bonding), we have extended our studies to 2- and 3-chloropyridine $\left(\mathrm{C}_{5} \mathrm{H}_{4} \mathrm{NCl}\right.$, Figure 1). The replacement of one of the hydrogen atoms in the parent pyridine molecule by a chlorine atom not only introduces two additional outer valence orbitals, due to the in-plane and out-of-plane orientations of the nominally atomic chlorine lone-pairs (LPs), but also perturbs the orbital 
manifold associated with the unsubstituted pyridine. It is also of interest to investigate how this modification to the electronic structure depends upon the substitution site.

In the present work, the third-order algebraic-diagrammatic construction [ADC(3)] approximation scheme $\mathrm{s}^{5-7}$ for the one-particle Green's function and the outer valence Green's function $(\mathrm{OVGF})^{7-9}$ methods have been employed to compute the valence shell ionization spectra of 2- and 3-chloropyridine. In general, the $\mathrm{ADC}(3)$ approach takes into account the physical effects, such as electron correlation in the initial and final states, and orbital relaxation, relevant to ionization. The calculated photoelectron spectra reproduce the main features observed in the corresponding experimental data and hence allow assignments to be made.

An interesting point to emerge from these calculations is that the states due to ionization of the nitrogen non-bonding ( $\sigma_{N} \mathrm{LP}$ ) orbital and the outermost ring $\pi$-orbital, which are widely separated at the HF level, merge together when more sophisticated theoretical methods are employed. The shift in the HF ionization energy is much larger for the $\sigma_{N}$ LP orbital than for the $\pi$-orbital. As a consequence of these non-uniform shifts, the $\sigma_{\mathrm{N}} \mathrm{LP}$ orbital changes position in the orbital sequence from being the highest occupied molecular orbital-2 (HOMO-2) to become HOMO-1.

In addition to modifying the electronic structure, the introduction of the chlorine atom may also affect the photoionization dynamics. These dynamics have been studied in the present work through measurements of the photoelectron anisotropy parameters $(\beta)$ and branching ratios (proportional to the photoionization partial cross sections). Valence orbital photoelectron angular distributions and branching ratios have been determined from their respective ionization thresholds up to a photon energy of $100 \mathrm{eV}$. As already mentioned, some of the molecular orbitals in the chloropyridines are predicted to correspond closely to atomic orbitals which are only slightly modified by the molecular environment. If a particular molecular orbital possesses a predominantly atomic character, then this should be reflected through the associated experimental observable. Thus, in chloropyridine it might be anticipated that the photoelectron anisotropy parameter for the $14 \mathrm{a}^{\prime}\left(\sigma_{\mathrm{N}} \mathrm{LP}\right)$ orbital exhibits an 
energy dependence similar to that calculated for the corresponding $\beta$-parameter due to the $\mathrm{N}$ $2 p$ atomic orbital. ${ }^{10}$ Similar expectations suggest that the photoionization dynamics of the two nominally halogen lone-pair orbitals in chloropyridine would show evidence of the Cooper minimum ${ }^{11,12}$ associated with the atomic $\mathrm{Cl} 3 \mathrm{p}$ orbital. ${ }^{13}$ The extent to which these atomic phenomena are observed depends upon the degree of atomic character retained by these molecular orbitals. Our experimentally determined photoelectron anisotropy parameters and branching ratios have been compared to the corresponding theoretical data calculated with the continuum multiple scattering (CMS - X $\alpha)$ approach. ${ }^{14}$

Previous experimental work on the photoelectron spectra of the chloropyridines is limited to HeI and HeII excited spectra of 2-chloropyridine ${ }^{15-18}$ and HeI excited spectra of 3chloropyridine. ${ }^{15,16}$ The ionization energy of the outermost orbital has also been measured using electron impact. ${ }^{19,20}$ All of these studies are restricted to the outer valence orbitals where the single particle model of ionization ${ }^{21}$ is expected to be valid. The inner valence region, where satellite structure may occur, has not been investigated.

\section{EXPERIMENTAL APPARATUS AND PROCEDURE}

Photoelectron spectra of chloropyridine were recorded using a rotatable hemispherical electron energy analyser and synchrotron radiation emitted by the Daresbury Laboratory storage ring. ${ }^{22}$ Detailed descriptions of the monochromator ${ }^{23}$ and the experimental procedure ${ }^{24}$ have been reported.

The photoionization differential cross section in the electric dipole approximation, assuming randomly oriented molecules and electron analysis in a plane perpendicular to the photon propagation direction, can be expressed in the form ${ }^{25}$

$$
\frac{\mathrm{d} \sigma}{\mathrm{d} \Omega}=\frac{\sigma_{\text {total }}}{4 \pi}\left[1+\frac{\beta}{4}(3 \mathrm{P} \cos 2 \theta+1)\right]
$$

where $\sigma_{\text {total }}$ is the angle integrated cross section, $\beta$ is the photoelectron anisotropy parameter, $\theta$ is the photoelectron ejection angle relative to the major polarization axis and $\mathrm{P}$ is the degree of linear polarization of the incident radiation. At each photon energy, photoelectron spectra 
were recorded at $\theta=0^{\circ}$ and $\theta=90^{\circ}$, thus allowing the anisotropy parameters and branching ratios to be determined once the polarization had been deduced. The degree of polarization was determined by recording Ar $3 p$ and $\mathrm{He} 1 \mathrm{~s}$ photoelectron spectra as a function of photon energy, and using the well-established $\beta$-parameters for these gases. ${ }^{25}$

The spectra were analysed by dividing the binding energy range into the regions specified in Table I, and the results are presented in the form of photoelectron anisotropy parameters and $\beta$-independent branching ratios. The branching ratio for a specific region (orbital) is defined as the intensity in that particular region divided by the sum of the intensity in all the energetically accessible regions. Our CMS - X $\alpha$ calculations for the branching ratios were performed for the outer valence orbitals only, that is, for photoelectron bands lying in energy regions $1-10$ (Table I). Therefore, to facilitate a meaningful comparison between experiment and theory, photoelectron intensity occurring in energy regions 11 and 12 has been ignored in deriving the experimental branching ratios (the measured branching ratios for regions $1-10$ sum to unity).

\section{COMPUTATIONAL DETAILS}

\section{A. ADC(3), OVGF and EOM-CC calculations}

Only a brief account will be given of the Green's function approach which we have used previously in similar studies of halogenated molecules. ${ }^{26-32}$ A detailed description has been provided recently by Trofimov et al. ${ }^{1}$ The ADC(3) approximation ${ }^{5-7}$ was employed to calculate the energies $(E)$ and (relative) spectral intensities (pole strengths, $P$ ) of the vertical transitions in 2- and 3-chloropyridine. This approximation describes the main "one-hole" $(1 h)$ and satellite "two-hole one-particle" $(2 h-1 p)$ electronic states through third and first order, respectively, in the residual electronic interaction, and is applicable in situations where the breakdown of the orbital picture of ionization ${ }^{21}$ takes place. The latter phenomenon manifests itself by a strong redistribution of spectral intensity from the main-lines to satellites, and often occurs for inner valence transitions. 
The carbon, nitrogen and chlorine K-shell orbitals and the chlorine L-shell orbital were kept frozen in the $\mathrm{ADC}(3)$ and OVGF calculations. The $\mathrm{ADC}(3)$ calculations were performed using the original code ${ }^{33}$ linked to the Gamess ab initio program package. ${ }^{34,35}$ The cc-pVDZ basis set ${ }^{36,37}$ with Cartesian representation of the d-functions was employed in the calculation of the spectral profiles. Theoretical photoelectron spectra were constructed from the $\mathrm{ADC}(3)$ results by convoluting the calculated data with Lorentzians of $0.4 \mathrm{eV}(\mathrm{FWHM})$.

For the OVGF calculations initially the cc-pVDZ basis set was used, allowing a direct comparison to be made with the $\mathrm{ADC}(3)$ results. Additionally, the ce-pVnZ $(n=D, T, Q, 5)$ and aug-cc-pVnZ ( $\mathrm{n}=\mathrm{D}, \mathrm{T}, \mathrm{Q})$ correlation consistent basis sets, ${ }^{36,37}$ with spherical representation of the d-functions, were then also used. These results from the OVGF calculations enabled the convergence of the ionization energies with respect to systematic improvements in the basis set to be studied, and they also allowed extrapolation to the complete basis set (CBS) limit. ${ }^{1}$

Finally, the outer valence vertical-ionization energies were computed using the equation-of-motion (EOM) coupled-cluster (CC) theory (EOM-IP-CC) at the singles and doubles (CCSD) level, ${ }^{38-41}$ as implemented in the Q-Chem program package. ${ }^{42}$ Also, the CC3 scheme (the CCSD model with approximate treatment of triple excitations) within the EOM-CC approach for excitation energies (EOM-EE-CC3) was employed in which the continuum orbifal approximation ${ }^{43}$ allows ionization energies to be evaluated. The EOM-EECC3 calculations were performed with the CFOUR program. ${ }^{44}$ The cc-pVTZ basis set ${ }^{36,37}$ was used in these calculations.

The ground state geometrical parameters of the chloropyridines for the above calculations of ionization energy were obtained through a full geometry optimization using second-order Møller-Plesset perturbation theory (MP2) together with the cc-pVTZ basis sets. The-MP2 calculations were carried out using the Gaussian package of programs. ${ }^{45}$

The Gaussian Cube utility ${ }^{45}$ was used to generate the total electron densities and the density differences between the RHF/ $\triangle \mathrm{SCF}$ and HF densities to show the effects of orbital relaxation for the cationic states. The HF densities were obtained as follows: the neutral 
ground-state HF orbitals were given as the initial guess for the cationic-state RHF calculations and the SCF procedure was terminated before the onset of the iterations. The GaussView program ${ }^{46}$ was employed to produce contour plots. The contour values suggested by GaussView, 0.001, 0.002, 0.004, 0.008, 0.02, 0.04, 0.08, 0.2, 0.4, 0.8, 2, were adopted. The Molden software ${ }^{47}$ was used to plot the orbitals computed at the HF/6-31G* level of theory.

The second-order perturbation theoretical approach (PT2) was employed to analyse the relaxation part of the ionization energy, ${ }^{48,49}$ as in our earlier study of pyridine. ${ }^{1}$ In this approach, the relaxation energy $\Delta E_{k}^{R}$ for the $k$-th orbital through second order is equal to one half of the screening energy $\Delta E^{S}(k)$ for that orbital: $\Delta E_{k}^{R}=\frac{1}{2} \Delta E^{S}(k)+O(3)$, where $O(3)$ denotes the remainder of perturbation series beyond second order. The screening energy $\Delta E^{S}(k)$ can be expanded in a perturbation series which starts in second order. ${ }^{48}$ The twoelectron Coulomb matrix elements in the numerators and the energy denominators of the distinct terms belonging to the second-order correction can be studied to obtain insight into the elementary processes that play a role in the screening of an electron vacancy and contribute to the relaxation energy ${ }^{1}$ (see supplementary material).

\section{B. CMS - Xa calculations of photoionization cross sections and photoelectron angular distributions}

Photoionization cross sections, $\sigma$, and angular distribution parameters, $\beta$, were calculated using the CMS - X $\alpha$ approach, following procedures that have been fully described $^{14}$ and used for a number of previous investigations of molecular halides. ${ }^{31,50}$ Briefly, model potentials were constructed using overlapping, spherical atomic volumes, placed at centres determined from the MP2/cc-pVTZ optimized geometry. Atomic sphere radii were estimated by the method of Norman, ${ }^{51}$ and scaled by a factor of 0.85 for all atoms, except chlorine for which a reduced factor of 0.83 was preferred. The spherical harmonic basis expansions for the initial neutral state were truncated at values, $l_{\max }$, of 5, 3, 2, and 1 in, respectively, the outer molecular region, the $\mathrm{Cl}$, the $\mathrm{C}$ and $\mathrm{N}$, and the $\mathrm{H}$ atom regions. The 
This manuscript was accepted by J. Chem. Phys. Click here to see the version of record. Version 20

trial functions were then iterated to achieve self-consistent ground state potentials in which the exchange contribution to an effective one-electron potential is represented using the $\mathrm{X} \alpha$ local density approximation. These potentials were subsequently adapted to have the correct asymptotic Coulombic form appropriate to solving the ion-photoelectron problem. Photoelectron wavefunctions, expanded in an enlarged basis of spherical harmonic functions (now 9, 5, 4, 3 for respectively outer, $\mathrm{Cl}, \mathrm{C}$ and $\mathrm{N}$, and $\mathrm{H}$ atom regions), and with radial terms obtained by direct numerical integration, were then obtained and used to construct the electric dipole photoionization matrix elements in an independent electron, frozen core approximation. Finally, values for $\sigma$ and $\beta$ were computed from these matrix elements, representing ionization of a fixed geometry, non-rotating, non-vibrating molecule.

\section{RESULTS AND DISCUSSION}

\section{A. Overview of electronic configuration and molecular orbital character}

Our HF calculations, performed in the $\mathrm{C}_{\mathrm{s}}$ molecular point group symmetry, predict the following ground state valence shell electronic configurations for 2-chloropyridine and 3chloropyridine:

$$
\begin{gathered}
\text { Inner valence : }\left(1 a^{\prime}\right)^{2}\left(2 a^{\prime}\right)^{2}\left(3 a^{\prime}\right)^{2}\left(4 a^{\prime}\right)^{2}\left(5 a^{\prime}\right)^{2}\left(6 a^{\prime}\right)^{2} \\
\text { Outer valence : }\left(7 a^{\prime}\right)^{2}\left(8 a^{\prime}\right)^{2}\left(9 a^{\prime}\right)^{2}\left(10 a^{\prime}\right)^{2}\left(11 a^{\prime}\right)^{2}\left(1 a^{\prime \prime}\right)^{2}\left(12 a^{\prime}\right)^{2}\left(2 a^{\prime \prime}\right)^{2}\left(13 a^{\prime}\right)^{2}\left(14 a^{\prime}\right)^{2} \\
\left(3 a^{\prime \prime}\right)^{2}\left(4 a^{\prime \prime}\right)^{2}
\end{gathered}
$$

where the core orbitals are omitted from the numbering scheme. The orbital character can be assessed from the Mulliken atomic populations ${ }^{52}$ given in Tables II and III, and the intercorrelation between these orbitals and those of pyridine is shown in Figure 2. The five highest occupied orbitals in each molecule are also plotted in Figure 3 using the results of our HF calculations.

As is normal for aromatic molecules, the molecular orbitals of the chloropyridines can be assigned as being of either $\sigma$ - or $\pi$-type. The aromatic system comprises three $\pi$-orbitals $\left(1 a^{\prime \prime}, 3 a^{\prime \prime}\right.$ and $\left.4 a^{\prime \prime}\right)$, with the inner-most $\pi$-orbital $\left(1 a^{\prime \prime}\right)$ in the chloropyridines being noticeably more tightly bound than the corresponding orbital $\left(1 b_{1}\right)$ in pyridine (Figure 2). Considerable 
admixtures of chlorine character are found in the $4 \mathrm{a}^{\prime \prime}$ and $1 \mathrm{a}^{\prime \prime}$ orbitals (Tables II and III, and Figure 3).

The $2 \mathrm{a}^{\prime \prime}$ orbital mainly corresponds to the chlorine lone-pair orientated perpendicular to the molecular plane (Figure 3). This orbital, formally of $\pi$-type, is, to some extent, delocalized over the ring atoms. The in-plane lone-pair orbital $\left(13 a^{\prime}\right)$ is of $\sigma$-type and is more strongly localized on the chlorine atom (Tables II and III, and Figure 3). The binding energies of these $\pi$ - and $\sigma$-type chlorine lone-pair orbitals are-lower in 2-chloropyridine than in 3-chloropyridine.

Of particular interest is the non-bonding $\sigma$-type lone-pair orbital $\left(14 \mathrm{a}^{\prime}\right)$ associated with the nitrogen atom (Figure 3). The Mulliken atomic populations (Tables II and III) indicate that although this orbital is mainly localized on the nitrogen atom, up to $\sim 28 \%$ and $\sim 7 \%$ of the electron density is delocalized over the carbon and hydrogen atoms, respectively. In 3chloropyridine, the $14 \mathrm{a}^{\prime}$ orbital contains a notable $(\sim 11 \%)$ admixture of chlorine character, although this admixture drops to $\sim 5 \%$ in 2-chloropyridine. The other orbitals in the chloropyridines are essentially $\sigma$-type bonding orbitals delocalised over the ring, hydrogen and chlorine atoms.

\section{B. Assignment of the photoelectron spectrum of 2-chloropyridine}

The present HF, OVGF, ADC(3), EOM-IP-CCSD and EOM-IP-CC3 results for the vertical outer valence ionization transitions of 2-chloropyridine, obtained with the cc-pVDZ and the aug-cc-cVTZ basis sets, are listed in Table IV, together with our experimental values. A basis set convergence study performed for these transitions at the OVGF level is documented in Table V. The $\mathrm{CC} 3$ ionization energies for the $\mathrm{CBS}$ limit $\left(\mathrm{CC} 3_{\infty}\right)$ were estimated by adding the difference between the results for the cc-pVoZ and the cc-pVTZ basis sets to the $\mathrm{CC} 3 / \mathrm{cc}-\mathrm{pVTZ}$ results.

At the HF level, the ionization energy of the nitrogen lone-pair state, $14 \mathrm{a}^{\prime}\left(\sigma_{\mathrm{N} \mathrm{LP}}\right)^{-1}$, places this state, as in pyridine, ${ }^{1}$ in the third position in the electronic sequence, above the $4 \mathrm{a}^{\prime \prime}(\pi)^{-1}$ and $3 \mathrm{a}^{\prime \prime}(\pi)^{-1}$ levels. When correlated methods are used, the ionization energy of the 
$14 \mathrm{a}^{\prime}$ orbital decreases by $\sim 1.5 \mathrm{eV}$, such that the nitrogen lone-pair state is shifted into the second lowest position in the theoretical spectrum. Although there is good agreement amongst the higher-order computational schemes in respect to the relative position of the $14 \mathrm{a}^{\prime}\left(\sigma_{\mathrm{N} \mathrm{LP}}\right)^{-1}$ state, the absolute ionization energies for this state and the other outer valence states vary strongly with both the method employed and the basis set used. Table V shows that the aug-cc-pVTZ basis set provides sufficiently converged results so that the OVGF, EOM-IP-CCSD and EOM-EE-CC3 ionization energies obtained with this basis should be fairly reliable.

Whilst the results from the OVGF and EOM-IP-CCSD methods are consistent with each other, the agreement with the experimental values is not entirely uniform. For example, discrepancies of up to $\sim 0.5 \mathrm{eV}$ are observed for the more tightly bound orbitals in the OVGF results for the CBS limit. Significantly more accurate predictions of the vertical ionization energies are obtained at the CC3 level of theory, both for the cc-pVTZ basis set and the CBS limit. Here, however, computational difficulties are encountered and do not allow the full set of outer valence ionization energies to be calculated.

All the theoretical methods, except CC3, considered in Table IV overestimate the splitting between the $4 \mathrm{a}^{\prime \prime}(\pi)^{-1}$ and the $14 \mathrm{a}^{\prime}\left(\sigma_{\mathrm{N}} \mathrm{LP}\right)^{-1}$ states, which, according to our experimental data, is $0.48 \mathrm{eV}$. Guided by our findings in pyridine, ${ }^{1}$ we expect that this discrepancy is due to an insufficient treatment of relaxation. Indeed, our $\triangle \mathrm{SCF} / \mathrm{cc}-\mathrm{pVTZ}$ calculations predict vertical ionization energies of 8.68 and $9.10 \mathrm{eV}$ for the $4 \mathrm{a}^{\prime \prime}(\pi)^{-1}$ and $14 \mathrm{a}^{\prime}\left(\sigma_{\mathrm{N}} / \mathrm{LP}\right)^{-1}$ states, respectively (Table VI). The resulting splitting of $0.42 \mathrm{eV}$ is in excellent agreement with the measured value. The relaxation energies of -0.82 and $-2.84 \mathrm{eV}$ in the $4 \mathrm{a}^{\prime \prime}(\pi)^{-1}$ and the $14 \mathrm{a}^{\prime}\left(\sigma_{\mathrm{NLP}}\right)^{-1}$ states, respectively, are similar to those in the unsubstituted pyridine molecule. ${ }^{1}$ This confirms again the key role of orbital relaxation in the ionization of the nitrogen lone-pair orbital. As can be seen in the density difference plots for 2chloropyridine (Figure 4), the relaxation patterns are almost the same as those for the corresponding cationic states of pyridine ${ }^{1}$ where a strong decrease of electron density occurs on the nitrogen atom, accompanied by moderate density increases on the hydrogen atoms, the $\mathrm{CH}$-bonds and two of the CC-bonds. However, an interesting new detail to emerge in the 
ionization of the nitrogen lone-pair in the halogenated species is that the chlorine atom is involved in the relaxation process. This can be seen from an increase of electron density in the areas near the chlorine $\sigma$-type lone-pair. As in pyridine, the $\pi$-system actively participates in the relaxation, and contributes, according to our PT2 analysis, up to $52 \%$ of the relaxation energy for the $\sigma_{\mathrm{N} L P}$ orbital ionization (Tables VI and S1). Since the orbitals are not redefined in this relaxation analysis, the electron density redistribution corresponding to the hole formation is effectively described by an orbital mixing that involves occupied and virtual orbitals, creating the relaxation effect. The main contribution gives the $3 \mathrm{a} "(\pi)-5 \mathrm{a} "\left(\pi^{*}\right)$ excitation, involving the highest occupied and the lowest unoccupied $\pi$-orbitals (Figures 3 and 5). The energetically more favorable $4 \mathrm{a}^{\prime \prime}(\pi)-6 \mathrm{a}^{\prime \prime}\left(\pi^{*}\right)$ excitation is only the second most important contribution (Table S1), apparently due to the less efficient overlap of the $4 \mathrm{a}^{\prime \prime}(\pi)$ and $6 \mathrm{a}^{\prime \prime}\left(\pi^{*}\right)$ orbitals with the $14 \mathrm{a}^{\prime}\left(\sigma_{\mathrm{NLP}}\right)$ MO. The relaxation of the electron in the $4 \mathrm{a}^{\prime \prime}(\pi)^{-1}$ and $3 \mathrm{a}^{\prime \prime}(\pi)^{-1}$ states is achieved mainly through various $\sigma-\sigma^{*}$ excitations (Table $\mathrm{S} 1$ ), so that the contribution of the $\pi-\pi^{*}$ excitations here is only $12-14 \%$ (Table VI).

The overall profile of the theoretical photoelectron spectrum of 2-chloropyridine (Figure 6) resembles that of unsubstituted pyridine, ${ }^{1}$ with the principal difference being the two distinct peaks, located near $12 \mathrm{eV}$, associated with the chlorine lone-pairs. In addition, small changes in the ionization energies of some of the cationic states, relative to those in pyridine, lead to a slightly modified appearance of certain band systems. In general, the agreement between the calculated profile and the experimental spectrum recorded at a photon energy of $80 \mathrm{eV}$ is satisfactory.

Figure 7 shows photoelectron spectra of 2-chloropyridine, recorded at a photon energy of $42.5 \mathrm{eV}$, for $\theta=0^{\circ}$ and $\theta=90^{\circ}$. These spectra show the outer valence bands in greater detail. They also illustrate the way in which angle resolved photoelectron spectra can help assign the type ( $\sigma, \pi$, or non-bonding) of orbital associated with a specific band even when the individual bands partially overlap. Ionization from three orbitals $\left(4 \mathrm{a}^{\prime \prime}(\pi), 14 \mathrm{a}^{\prime}\left(\sigma_{\mathrm{N}} \mathrm{LP}\right)\right.$ and $3 \mathrm{a}^{\prime \prime}(\pi)$ ) contribute to the spectrum in the binding energy range $\sim 9$ to $11 \mathrm{eV}$, and three peaks are observed with maxima at $9.63,10.11$ and $10.75 \mathrm{eV}$. It is noticeable that in the spectrum recorded at $\theta=0^{\circ}$ the peaks at 9.63 and $10.75 \mathrm{eV}$ are more intense than that at $10.11 \mathrm{eV}$, 
whilst the opposite behavior is evident in the spectrum recorded at $\theta=90^{\circ}$. This relative variation in peak intensity is a consequence of the $\beta$-parameter of an electron ejected from a $\pi$-type orbital being much higher than that of an electron ejected from a $\sigma$-type orbital at a photon energy of $42.5 \mathrm{eV} .54$

The experimental peak maxima values for the three outermost orbitals are consistent with the results obtained in our EOM-IP-CCSD and EOM-EE-CC3 calculations (Table IV). The splitting between the ionization energies for the $4 \mathrm{a}^{\prime \prime}$ and $3 \mathrm{a}^{\prime \prime}$ orbitals $(1.19 \mathrm{eV})$ obtained in the $\mathrm{ADC}(3)$ calculations is also in accord with the experimental value $(1.12 \mathrm{eV})$, but the ADC(3) calculations overestimate the splitting between the $4 a^{\prime \prime}$ and $14 a^{\prime}$ orbitals and this leads to a discrepancy between the theoretical and experimental spectra plotted in Figure 6.

The next two peaks, with maxima at 11.56 and $12.11 \mathrm{eV}$ in the experimental spectrum, correspond to ionization from the chlorine lone-pair orbitals $13 \mathrm{a}^{\prime}\left(\sigma_{\mathrm{Cl} L \mathrm{LP}}\right)$ and $2 \mathrm{a}^{\prime \prime}\left(\pi_{\mathrm{Cl} L \mathrm{LP}}\right)$, respectively. Figure 7 shows that at a photon energy of $42.5 \mathrm{eV}$, an energy which lies close to that of the predicted $\mathrm{Cl} 3 \mathrm{p}$ Cooper minimum, ${ }^{13}$ the $\beta$-parameter for the $13 \mathrm{a}^{\prime}$ orbital is much lower than that for the $2 \mathrm{a}^{\prime \prime}$ orbital. This difference in $\beta$-values suggests that the $2 \mathrm{a}^{\prime \prime}$ orbital, which is orientated perpendicular to the molecular plane, interacts with the $\pi$-type orbitals on the carbon ring atoms. Such an interpretation is supported by the Mulliken atomic populations (Table II) and by the molecular orbital plots in Figure 3 which indicate that the $13 \mathrm{a}^{\prime}$ orbital may be considered as an essentially atomic $3 \mathrm{p}$ chlorine orbital lying in the molecular plane whereas the $2 \mathrm{a}^{\prime \prime}$ orbital contains contributions from the carbon atoms.

Our calculations reproduce the portion of the photoelectron spectrum containing the two peaks associated with the chlorine lone-pairs quite accurately. The agreement between the OVGF and the EOM-IP-CCSD calculations (using the aug-cc-pVTZ basis set) is very good (Tables IV and V). As expected, the EOM-EE-CC3 results demonstrate the best overall agreement with experiment.

According to our PT2 analysis (Table VI), the relaxation shifts for the $13 \mathrm{a}^{\prime}\left(\sigma_{\mathrm{Cl} L \mathrm{LP}}\right)$ and $2 \mathrm{a} "\left(\pi_{\mathrm{Cl} L \mathrm{LP}}\right)$ orbitals are -1.88 and $-0.95 \mathrm{eV}$, respectively. Since only the lowest state of each symmetry can be treated within the $\triangle \mathrm{SCF}$ framework, the relaxation energy here was 
estimated using only the PT2 approach. ${ }^{1,48,49}$ The relevance of the PT2 predictions is supported by the fair agreement between the PT2 results for the $4 \mathrm{a}^{\prime \prime}(\pi)^{-1}$ and $14 \mathrm{a}^{\prime}\left(\sigma_{\mathrm{N}} \mathrm{LP}\right)^{-1}$ states and the results of the $\triangle \mathrm{SCF}$ calculations (Table VI).

The computed relaxation shifts for the $13 \mathrm{a}^{\prime}\left(\sigma_{\mathrm{Cl} L P}\right)$ and $2 \mathrm{a}^{\prime \prime}\left(\pi_{\mathrm{Cl} L \mathrm{LP}}\right)$ orbitals are substantially smaller than that for the nitrogen lone-pair orbital. The PT2 analysis (Table S1) allows the differences in the relaxation mechanisms to be understood. For ionization of the $13 \mathrm{a}^{\prime}\left(\sigma_{\mathrm{Cl} L \mathrm{LP}}\right)$ orbital, the principle relaxation contribution comes from the $2 \mathrm{a}\left(\pi_{\mathrm{Cl} L P}\right)-8 \mathrm{a}^{\prime \prime}$ mixing. Remarkably, the $8 \mathrm{a}^{\prime \prime}$ orbital can be identified rather clearly as a p-type atomic orbital of chlorine (Figure 5) and thus can be viewed as the chlorine $4 p$ Rydberg orbital. The ionization is strongly chlorine localized, which leads to significant coupling between the $\sigma_{\mathrm{Cl}} \mathrm{LP}$ charge and the electron density of the chlorine localized $\pi_{\mathrm{Cl} L \mathrm{LP}}$ and $4 \mathrm{p}$ orbitals, and to a large absolute value of the Coulomb integral ( $\left.\sigma_{\mathrm{Cl} L \mathrm{LP}} \sigma_{\mathrm{Cl} L \mathrm{LP}} \mid \pi_{\mathrm{Cl} L \mathrm{LP}} \mathrm{Cl} 14 \mathrm{p}\right) \equiv$ $\left\langle\pi_{\mathrm{ClLP}}\left|\hat{J}_{\sigma \mathrm{Cl} L \mathrm{P}}\right| \mathrm{Cl} 4 \mathrm{p}\right\rangle$. The localization factors here outweigh the energetically unfavorable accessibility of the $\mathrm{Cl} 4 \mathrm{p}$ orbital, which is separated from the $2 \mathrm{a} "\left(\pi_{\mathrm{Cl}} \mathrm{LP}\right)$ orbital by an energy gap of $30 \mathrm{eV}$ (Table S1). The more accessible $\pi-\pi^{*}$ excitations, characterized by energy gaps of $\sim 12 \mathrm{eV}$, are less important because the stronger spatial delocalization of the $\pi$-MOs reduces the magnitude of the Coulomb matrix elements $\left\langle\pi\left|\hat{J}_{\sigma \mathrm{Cl} L P}\right| \pi^{*}\right\rangle$ (Table S1). This is in contrast to the relaxation accompanying the ionization of nitrogen lone-pair where $\pi$ - $\pi^{*}$ excitations play the main role.

The complex structure appearing in the experimental spectrum in the binding energy range $\sim 12.5-15.5 \mathrm{eV}$ is similar to that in the corresponding portion in the spectrum of unsubstituted pyridine. ${ }^{1}$ The maxima observed at 13.03, 13.91 and $14.79 \mathrm{eV}$ can be assigned to ionization of three $\sigma$-type orbitals, namely $12 \mathrm{a}^{\prime}, 11 \mathrm{a}^{\prime}$ and $10 \mathrm{a}^{\prime}$, respectively. Ionization of the $1 \mathrm{a}^{\prime \prime}(\pi)$ orbital contributes to the peak at $13.91 \mathrm{eV}$. This transition is strongly affected by satellite formation. The intensity of the $1 \mathrm{a}^{\prime \prime}(\pi)^{-1}$ main-line (located at $14.09 \mathrm{eV}$ according to our $\mathrm{ADC}(3)$ calculations) is only 0.56 , which means that nearly half of the intensity associated with this transition is transferred to various satellites. One of these satellites, having a spectral intensity of 0.09 , is predicted at $13.92 \mathrm{eV}$ and therefore occurs at an ionization energy slightly less than that of the $1 \mathrm{a}^{\prime \prime}(\pi)^{-1}$ main-line. Thus, this is an example of 
a shake-down satellite. Satellites of this nature are rarely encountered in organic molecules at such low binding energies. For this satellite, the dominant $2 \mathrm{~h}-1 \mathrm{p}$ configurations are of the $4 \mathrm{a}^{\prime \prime}(\pi)^{-2} \pi^{*}$ and $4 \mathrm{a}^{\prime \prime}(\pi)^{-1} 3 \mathrm{a}(\pi)^{-1} \pi^{*}$ type. Interestingly, there is also a $1 \%$ intensity contribution associated with the virtual $6 a^{\prime \prime}\left(\pi^{*}\right)$ orbital. This contribution is due to ground state correlation. According to our calculations, various shake-up satellites deriving intensity from the $10 \mathrm{a}^{\prime}(\sigma)^{-1}$ and $11 \mathrm{a}^{\prime}(\sigma)^{-1}$ main-lines are also located in the spectral range under consideration and contribute to the width of the observed photoelectron bands.

Experimentally, the contributions from the $1 \mathrm{a}^{\prime \prime}(\pi)$ and $11 \mathrm{a}^{\prime}(\sigma)$ orbitals overlap to form the band with a maximum observed at $13.91 \mathrm{eV}$ in the spectrum recorded at $\theta=0^{\circ}$, and at $13.98 \mathrm{eV}$ in the spectrum recorded at $\theta=90^{\circ}$. The direction of this shift is consistent with the ionization energy of the $1 \mathrm{a}^{\prime \prime}$ orbital being slightly lower than that of the $11 \mathrm{a}^{\prime}$ orbital, in agreement with the $\operatorname{ADC}(3)$ predictions. The contribution from the $1 \mathrm{a}^{\prime \prime}(\pi)$ orbital to the photoelectron peak is confirmed by the high $\beta$-value measured at a photon energy of $42.5 \mathrm{eV}$ compared to the lower $\beta$-value for the neighbouring peak at a binding energy of $13.03 \mathrm{eV}$, due solely to the $12 \mathrm{a}^{\prime}(\sigma)$ orbital. ${ }^{5}$

The next section of the photoelectron spectrum, between $\sim 15.5$ and $18.5 \mathrm{eV}$, is reproduced well by our calculations. Three peaks, centred at $15.83,16.23$ and $17.23 \mathrm{eV}$, due to ionization of the $9 a^{\prime}, 8 a^{\prime}$ and $7 a^{\prime}$ orbitals, respectively, are superimposed upon a dense, low intensity background attributed to shake-up satellites.

At slightly higher binding energy, a small peak appears in the experimental spectrum at $\sim 18.8 \mathrm{eV}$. The possibility that this peak is due to an impurity in the sample cannot be excluded but no other structure which might also be attributed to an impurity is discernible. This peak is noticeably more intense in the spectrum recorded at $\theta=0^{\circ}$ than in that recorded at $\theta=90^{\circ}$. Thus, it is associated with a significantly positive $\beta$-value, such as that expected for an electron ejected from a $\pi$-type orbital at a photon energy of $42.5 \mathrm{eV}$. Qualitatively, our calculations reproduce this feature, although not in terms of a single dominant transition. A cluster of low intensity shake-up satellites, mainly associated with the $1 a^{\prime \prime}(\pi)$ orbital, are predicted in this region, and collectively form a small peak in the theoretical spectrum. 
At binding energies above $20 \mathrm{eV}$ the experimental spectrum becomes increasingly diffuse. According to our theoretical results this reflects the breakdown regime of the single particle model of ionization where extended groups of overlapping satellite states replace the dominant main-lines transitions. The structure in this region is extremely complex, as can be seen in the bar spectrum under the theoretical envelope shown in Figure 6. Interestingly, some of the more pronounced features in the experimental spectrum can still be reproduced surprisingly well by the theoretical envelope generated from the seemingly erratic satellite manifold. For example, the broad doublet with maxima observed at 19.7 and $20.4 \mathrm{eV}$ is predicted to be formed by states deriving their intensity from ionization of the $6 a^{\prime}(\sigma)$ and $5 \mathrm{a}^{\prime}(\sigma)$ electrons.

At higher energy, the interpretation of the experimental spectrum, showing three broad peaks centred at $\sim 23,25$ and $29 \mathrm{eV}$, based upon the theoretical results becomes less conclusive as the theoretical spectral profile looks more structured. However, it can be expected that the peaks at 23 and $29 \mathrm{eV}$ are mainly due to transitions related to the $4 \mathrm{a}\left({ }^{\prime} \sigma\right)$ and $1 \mathrm{a}^{\prime}(\sigma)$ orbitals, respectively, while the peak centred at $25 \mathrm{eV}$ is formed by states deriving intensity from the $3 \mathrm{a}\left({ }^{\prime} \sigma\right)$ and $2 \mathrm{a}^{\prime}(\sigma)$ ionization.

\section{Assignment of the photoelectron spectrum of 3-chloropyridine}

The photoelectron spectrum of 3-chloropyridine (Figure 8) qualitatively resembles that of 2-chloropyridine (Figure 6) and will therefore be discussed more briefly. The correlation diagram for the outer valence orbitals (Figure 2) shows that the only major difference between the two isomers concerns the $11 \mathrm{a}^{\prime}(\sigma)$ and $1 \mathrm{a}^{\prime \prime}(\pi)$ orbitals which exchange positions in the orbital sequence. In 3-chloropyridine, $1 \mathrm{a}^{\prime \prime}(\pi)$ is the less tightly bound orbital. All our theoretical results are listed in Tables VII and VIII, together with the photoelectron band maxima estimated from the present experimental spectra. In general, all the ionization energies obtained using the correlated methods and basis sets reported in Tables VII and VIII provide a consistent description of the outer valence ionization. In particular, the EOM-EE$\mathrm{CC} 3$ results are in excellent agreement with the measurements. 
The outermost photoelectron band in the experimental spectrum of 3-chloropyridine (Figure 9), recorded at a photon energy of $42.5 \mathrm{eV}$, is due to ionization of the $4 \mathrm{a}^{\prime \prime}(\pi)$ and $14 \mathrm{a}^{\prime}\left(\sigma_{\mathrm{N}} \mathrm{LP}\right)$ orbitals. The calculated ionization energies of these two orbitals are similar (Tables VII and VIII), and hence the individual contributions overlap. Nevertheless, the change in the peak shape in the spectra recorded at $\theta=0^{\circ}$ and $\theta=90^{\circ}$ (Figure 8) indicates that the $4 \mathrm{a}^{\prime \prime}(\pi)$ orbital has the lower ionisation energy, in accord with the theoretical predictions (Tables VII and VIII). In the spectrum recorded at $\theta=0^{\circ}$ the peak maximum occurs at $9.58 \mathrm{eV}$ and a shoulder is observed to higher binding energy, whilst in that recorded at $\theta=90^{\circ}$ the maximum shifts to $9.83 \mathrm{eV}$ and the shoulder is observed to lower binding energy. This change in the photoelectron band profile is a consequence of the $\beta$-value for an electron ejected from a $\pi$-orbital differing significantly from that for an electron ejected from a $\sigma$-orbital at a photon energy of $42.5 \mathrm{eV} .54$

Our calculations overestimate the splitting between the $4 \mathrm{a}^{\prime \prime}(\pi)^{-1}$ and $14 \mathrm{a}^{\prime}\left(\sigma_{\mathrm{N} L P}\right)^{-1}$ transitions, and this results in two separate peaks appearing in the theoretical photoelectron spectrum (Figure 8). The $\mathrm{ADC}(3)$ calculations underestimate the relaxation effects in the $14 \mathrm{a}^{\prime}\left(\sigma_{\mathrm{N}} \mathrm{LP}\right)^{-1}$ state, so an improved agreement between experiment and theory can be expected upon a more satisfactory treatment of relaxation. The orbital relaxation energies of -0.83 and $-2.74 \mathrm{eV}$ in the $4 \mathrm{a}^{\prime \prime}(\pi)^{-1}$ and $14 \mathrm{a}^{\prime}\left(\sigma_{\mathrm{N}} \mathrm{LP}\right)^{-1}$ states, respectively, of 3-chloropyridine, obtained at the $\triangle \mathrm{SCF}$ level of theory (Table VI), are similar to those in 2-chloropyridine and pyridine. ${ }^{1}$ The relaxation induced density redistribution (Figure 10) shows the familiar pattern, as in Figure 4 .

Ionization of the $3 \mathrm{a}^{\prime \prime}(\pi)$ orbital gives rise to a band with a maximum observed at 10.60 $\mathrm{eV}$, in accord with the theoretical predictions. The results from our ADC(3), OVGF, EOMIP-CCSD and EOM-EE-CC3 calculations give a splitting between the ionization energies of the $4 a^{\prime \prime}(\pi)$ and $3 \mathrm{a}^{\prime \prime}(\pi)$ orbitals which is in good agreement with the measured value $(1.02 \mathrm{eV})$.

The next two peaks in the photoelectron spectrum are due to the chlorine lone-pairs $\left(13 \mathrm{a}^{\prime}\left(\sigma_{\mathrm{Cl} L P}\right)\right.$ and $\left.2 \mathrm{a}^{\prime \prime}\left(\pi_{\mathrm{Cl} L P}\right)\right)$. It is noticeable from the Mulliken populations in Tables II and 
III and MO plots in Figure 3 that the contribution from the nitrogen atom to the $2 \mathrm{a}^{\prime \prime}\left(\pi_{\mathrm{Cl} L \mathrm{LP}}\right)$ is substantially larger in 3-chloropyridine than in 2-chloropyridine.

More specifically, the nitrogen character of the $2 \mathrm{a}^{\prime \prime}\left(\pi_{\mathrm{Cl} L \mathrm{LP}}\right)$ orbital in 3-chloropyridine is larger, by about $10 \%$, compared to that in 2-chloropyridine. Interestingly, the nitrogen character of the $14 \mathrm{a}^{\prime}\left(\sigma_{\mathrm{N}} \mathrm{LP}\right) \mathrm{MO}$ also increases by about $5 \%$. At the same time, the $\pi_{\mathrm{Cl}} \mathrm{LP}$ and $\sigma_{N}$ LP MOs of 3-chloropyridine lose about the same amount of their chlorine character (10 and $3 \%$, respectively). Also, the chlorine character in the nitrogen lone-pair orbital $14 \mathrm{a}^{\prime}\left(\sigma_{\mathrm{N}} \mathrm{LP}\right)$ of 3 -chloropyridine increases by about 5\%. Thus, all lone-pair orbitals in 3-chloropyridine appear to be more delocalized than the corresponding orbitals in 2-chloropyridine. This is consistent with the systematically smaller relaxation shifts obtained for ionization of these orbitals in 3-chloropyridine at the PT2 level (Table VI). We recall that the relaxation shift depends on the magnitude of the $\left\langle\varphi_{i}\left|\hat{J}_{\varphi \mathrm{LP}}\right| \varphi_{a}\right\rangle$ integral and is therefore more pronounced for compact orbitals, being largest for K-shell ionization processes.

It should also be noted that all the theoretical methods predict ionization energies of the chlorine lone-pairs that are larger in 3-chloropyridine than in 2-chloropyridine, in agreement with the experimental results (Tables IV and VII). The opposite behavior occurs for the energy levels of the nitrogen lone-pair orbitals $\sigma_{N}$ LP. Our calculations employing the natural bond orbital (NBO) ${ }^{55-58}$ analysis of the HF/cc-pVDZ wavefunctions provide an explanation for these differences. According to our results, the NBO donor-acceptor interaction of the nitrogen lone-pair orbital $\sigma_{\mathrm{N}} \mathrm{LP}$ with the $\sigma *$-type $\mathrm{C}$ - $\mathrm{Cl}$ antibonding orbital $\left(\sigma_{\mathrm{N}} \mathrm{LP} \rightarrow \sigma^{*} \mathrm{C}-\mathrm{Cl}\right)$ amounts to 0.6 and $6.7 \mathrm{kcal} / \mathrm{mol}$ for 3-chloropyridine and 2-chloropyridine, respectively. This large difference shows clearly that the nitrogen lone-pair which stabilizes the vacancy on chlorine in the position "2", has practically no effect on the chlorine in the position "3", which is an easily understood topological effect. Moreover, our NBO calculations predict a substantial electron density transfer from the $\sigma_{\mathrm{N}} \mathrm{LP}$ orbital to a Rydberg-type orbital located at the $\mathrm{C}_{2}$ site in 2-chloropyridine $(\sim 7.8 \mathrm{kcal} / \mathrm{mol})$ which should also stabilize the states with chlorine vacancies. In contrast, the latter effect is completely missing in 3-chloropyridine. The same active donor role of the $\sigma_{\mathrm{N}} \mathrm{LP}$ orbital in 2-chloropyridine explains the increase in the ionization energy of this orbital in 2-chloropyridine compared to its energy in unsubstituted 
pyridine and 3-chloropyridine (Figure 2). The present NBO calculations show that in the above electron density transfer processes the unoccupied orbitals localized at the chlorine site play an acceptor role. This can probably be interpreted as a manifestation of the negative inductive effect of chlorine $(-I)$.

The calculated relaxation shifts for the $13 \mathrm{a}^{\prime}\left(\sigma_{\mathrm{Cl} L P}\right)$ and $2 \mathrm{a}^{\prime \prime}\left(\pi_{\mathrm{Cl} L \mathrm{LP}}\right)$ orbitals are -1.44 and $-0.91 \mathrm{eV}$, respectively (Table VI). As in 2-chloropyridine, the chlorine lone-pair relaxation shifts are smaller than that of the nitrogen lone-pair, which-again can be attributed to the different relaxation mechanism based on the screening of the chlorine vacancy by promotions of the following type: $\pi_{\mathrm{Cl} L \mathrm{LP}-\mathrm{Cl}} 4 \mathrm{p}$ and $\sigma_{\mathrm{Cl} L \mathrm{LP}-\sigma^{*}}$ (Tables S1).

Ionization of the $1 \mathrm{a}^{\prime \prime}(\pi)$ and $11 \mathrm{a}^{\prime}(\sigma)$ orbitals provides a good demonstration of the way in which angle resolved photoelectron spectra allow the type of orbital to be identified even though the associated bands overlap to some extent. In the spectrum recorded at $\theta=0^{\circ}$, a band with a maximum at $13.75 \mathrm{eV}$ is observed, together with a shoulder on the high binding energy side. In contrast, in the spectrum recorded at $\theta=90^{\circ}$, two peaks are observed with the higher energy band having a maximum at $14.17 \mathrm{eV}$. Such angle dependent variations show that the peak at $13.75 \mathrm{eV}$ is due to the $1 \mathrm{a}^{\prime \prime}(\pi)$ orbital and that at $14.17 \mathrm{eV}$ to the $11 \mathrm{a}^{\prime}(\sigma)$ orbital.

Our $\operatorname{ADC}(3)$ calculations predict that ionization of the $1 \mathrm{a}^{\prime \prime}(\pi)$ orbital gives rise to a large number of satellites (labelled 7 in Figure 8) which lie on the high binding energy side of the associated main-line (having a pole strength of only 0.5 ).

The photoelectron bands centred at 15.76, 16.24 and $17.16 \mathrm{eV}$ are reproduced satisfactorily in the theoretical spectrum and may be assigned to the $9 a^{\prime}(\sigma)^{-1}, 8 a^{\prime}(\sigma)^{-1}$ and $7 a^{\prime}(\sigma)^{-1}$ transitions, respectively. The substantial width of the experimental peaks is most likely due to the presence of numerous low intensity satellites.

As in 2-chloropyridine, a small peak is observed in the experimental spectrum at a binding energy of $\sim 18.7 \mathrm{eV}$. Although a dense manifold of satellites related to the $1 \mathrm{a}^{\prime \prime}(\pi)$ ionization appears in the theoretical spectrum, the spectral envelope does not result in a discernible peak. This could be due to subtleties in the distribution of the satellites not 
adequately described by our computations. Alternatively, the peak could be due to a component of the $6 \mathrm{a}^{\prime}(\sigma)^{-1}$ ionization manifold which is placed at too high an energy by our calculations.

According to our calculations, above $19 \mathrm{eV}$ and beginning with the $6 \mathrm{a}^{\prime}(\sigma)$ ionization, the one-particle picture of ionization becomes invalid. ${ }^{21}$ This inner valence region of the photoelectron spectrum of 3-chloropyridine will not be considered in detail as it is similar to that of 2-chloropyridine. A comparison between the experimental and theoretical spectra (Figure 8) allows an approximate interpretation of the inner valence structure to be obtained.

\section{Photoelectron angular distributions and branching ratios}

The experimental and theoretical photoelectron anisotropy parameters and branching ratios for the valence orbitals of 2-chloropyridine are plotted in Figures 11 and 12, respectively. The corresponding data for 3-chloropyridine are similar and are available in supplementary material. It appears that the substitution site for the chlorine atom has little effect on the photoionization dynamics, at least as monitored by the photoelectron $\beta$ parameters and branching ratios.

Binding energy region 1 (Table I) encompasses contributions from the $4 a^{\prime \prime}(\pi)$ and $14 \mathrm{a}^{\prime}\left(\sigma_{\mathrm{N}} \mathrm{LP}\right)$ orbitals, although the two components are not resolved experimentally. The calculated $\beta$-parameter for the $4 \mathrm{a}^{\prime \prime}(\pi)$ orbital rises rapidly from a low value at threshold to reach a plateau value of $\sim 1.5$ at a photon energy of $50 \mathrm{eV}$. Such an energy dependent behavior is typical of that observed and predicted ${ }^{30-32,50,59}$ for electron ejection from a $\pi$ orbital. The calculated value of the anisotropy parameter due to the $14 \mathrm{a}^{\prime}\left(\sigma_{\mathrm{N}} \mathrm{LP}\right)$ orbital rises more slowly as the energy increases. This calculated increase in the $\beta$-value for the $14 \mathrm{a}^{\prime}$ orbital is also slower than that predicted for the anisotropy parameter associated with the nitrogen $2 \mathrm{p}$ electron. ${ }^{10}$ The Mulliken atomic populations for the $14 \mathrm{a}^{\prime}$ orbital (Table II) provide a plausible means of reconciling this disparity. The populations show that the $14 \mathrm{a}^{\prime}$ orbital contains significant contributions from the carbon atoms, and thus should not be considered simply as an unmodified nitrogen lone-pair. 
The photoelectron bands due to the $13 \mathrm{a}^{\prime}\left(\sigma_{\mathrm{Cl} L P}\right)$ and $2 \mathrm{a}^{\prime \prime}\left(\pi_{\mathrm{Cl} L \mathrm{LP}}\right)$ orbitals lie in energy regions 3 and 4, respectively. These two orbitals may be considered as essentially chlorine lone-pairs and hence their associated photoionization dynamics would be expected to be influenced by the atomic $\mathrm{Cl} 3 \mathrm{p}$ Cooper minimum which occurs at a photon energy of $\sim 42$ eV. ${ }^{13}$ The dip due to this atomic phenomenon is clearly observed in the $\beta$-parameter for the $13 \mathrm{a}^{\prime}$ orbital, indicating that this molecular orbital retains its atomic properties to a large extent even within the molecular environment. Note the high contribution from the chlorine atom in the Mulliken atomic populations for the $13 \mathrm{a}^{\prime}$ orbital (Table $\mathrm{II}$ ). In the minimum, the experimental $\beta$-value for this orbital is $\sim 0.4$, which is only slightly greater than the theoretical prediction $(\sim-0.2)$ for atomic chlorine. ${ }^{13}$ The calculated $\beta$-parameter for the $13 \mathrm{a}^{\prime}$ orbital also exhibits a dip due to the Cooper minimum, although its position is shifted slightly to lower energy compared to experiment. For the other nominally chlorine lone-pair orbital $\left(2 \mathrm{a}^{\prime \prime}\right)$, the influence of the Cooper minimum appears only weakly in the experimental data and in the theoretical curve it is almost absent. It is noticeable that the Mulliken atomic populations for the $2 \mathrm{a}^{\prime \prime}$ orbital show increased contributions from the $\mathrm{C}_{4}, \mathrm{C}_{5}$ and $\mathrm{C}_{6}$ atoms, together with a reduced contribution from the $\mathrm{Cl}$ atom, compared to those for the $13 \mathrm{a}^{\prime}$ orbital. Moreover, as the $2 \mathrm{a}^{\prime \prime}\left(\pi_{\mathrm{Cl} L \mathrm{LP}}\right)$ orbital is orientated perpendicular to the molecular plane it may interact more readily with the ring $\pi$-orbitals and hence loose much of its atomic character. This reduction in the $\mathrm{Cl} 3 \mathrm{p}$ character of the $2 \mathrm{a}^{\prime \prime}$ orbital is consistent with the dip due to the chlorine atom Cooper minimum being strongly attenuated in the anisotropy parameter.

The experimental and theoretical $\beta$-parameters for the $12 \mathrm{a}^{\prime}$ orbital exhibit the energy dependence expected for an electron ejected from a $\sigma$-type orbital. ${ }^{30-32,50,54}$ In contrast, the $\beta$ parameter measured for region 7 , which encompasses the photoelectron band due to the $10 \mathrm{a}^{\prime}(\sigma)$ orbital, is much higher than predicted. This discrepancy may be due to satellites, calculated to lie within the same binding energy range as that spanned by the $10 \mathrm{a}^{\prime}(\sigma)^{-1}$ photoelectron band, associated with the $1 a^{\prime \prime}(\pi)$ orbital. Such satellites would be expected to have high $\beta$-values. Therefore, photoelectron intensity arising from these satellites would tend to increase the anisotropy parameter for region 7 above the value due solely to the $10 \mathrm{a}^{\prime}$ orbital. 
Figure 12 shows that a satisfactory agreement is obtained between the experimental and theoretical branching ratios for binding energy regions $1-10$. The calculated ratios exhibit some structure at low photon energies that is not evident in the experimental data. This structure is partly due to predicted near-threshold resonances which give rise to intense peaks in some of the calculated photoionization partial cross sections (not shown). Another contributing factor to this structure is associated with the many ionization thresholds which occur in the binding energy range $\sim 10-20 \mathrm{eV}$ (Table IV). As each threshold is crossed, abrupt changes tend to appear in the branching ratios derived from the partial cross sections.

The branching ratios for the $13 \mathrm{a}^{\prime}\left(\sigma_{\mathrm{Cl} L P}\right)$ and the $2 \mathrm{a}^{\prime \prime}\left(\pi_{\mathrm{Cl}} \mathrm{LP}_{\mathrm{P}}\right)$ orbitals display a dip at a photon energy of $\sim 40 \mathrm{eV}$ due to the $\mathrm{Cl} 3 \mathrm{p}$ Cooper minimum. The correspondence between the experimental and theoretical results is good although the predicted minimum occurs a few $\mathrm{eV}$ lower than the observed position. Interestingly, the influence of the Cooper minimum is clearly evident in the branching ratios for both of the nominally chlorine lone-pair orbitals whereas its effect on the corresponding $\beta$-parameters is only noticeable in that associated with the $13 \mathrm{a}^{\prime}$ orbital (Figure 11). Such behavior is consistent with that observed in similar studies on chlorobenzene ${ }^{60}$ where the influence of the Cooper minimum was apparent in the branching ratios associated with the in-plane and the out-of-plane orientations of the chlorine lone-pair orbitals, but its effect on the anisotropy parameters was only evident in that of the in-plane orientation.

\section{SUMMARY}

The valence shell electronic structure of 2-chloropyridine and 3-chloropyridine has been investigated both theoretically, by using various approaches to calculate ionization energies and spectral intensities, and experimentally by employing synchrotron radiation to record angle resolved photoelectron spectra. Although the photoelectron spectra of the two isomers are generally similar, the substitution site for the chlorine atom produces subtle shifts in orbital ionization energies, which, in turn, modify specific band systems. The ADC(3) method has been employed to generate the complete valence shell photoelectron spectrum of 
each isomer. The theoretical profiles are in good accord with the experimental spectra and have allowed most of the observed structure to be assigned. The calculations show that the single particle model of ionization becomes invalid at binding energies above $\sim 19 \mathrm{eV}$. In the inner valence region, electron correlation leads to a reduction in main-line pole strength and to a corresponding increase in satellite formation.

The OVGF method has been employed to study the convergence of the results to the CBS limit. The EOM-IP-CCSD and EOM-EE-CC3 computational schemes have been used to obtain accurate estimates of the vertical ionization energies for the outer valence shell. In particular, the CC3 results obtained with the cc-pVTZ basis set and in the CBS limit are in excellent agreement with the measurements. The theoretical work has enabled the influence of electron correlation and orbital relaxation on the orbital ionization energies to be examined in detail. The relaxation mechanism for the oN LP orbital has been found to differ from that for the $\sigma_{C l}$ LP orbital. For the $\sigma_{\mathrm{N} L P}$ orbital, $\pi-\pi^{*}$ excitations play the main role in the screening of the lone-pair hole, whereas for the $\sigma_{\mathrm{Cl}} \mathrm{LP}$ orbital excitations localized at the chlorine site involving the chlorine $\pi_{\mathrm{Cl}} \mathrm{LP}$ lone-pair and the $\mathrm{Cl} 4 \mathrm{p}$ Rydberg orbital are the most important. For the $\pi_{\mathrm{Cl} L \mathrm{LP}}$ orbital, the main relaxation contribution comes from the $\sigma_{\mathrm{Cl}} \mathrm{LP}-\sigma^{*}$ excitations, where $\sigma^{*}$ is a high energy Rydberg-type orbital.

Photoelectron anisotropy parameters and branching ratios have been derived from the angle resolved photoelectron spectra and compared to the corresponding predictions obtained through the CMS - X $\alpha$ approach. The influence of the chlorine atom Cooper minimum on the $\beta$-parameter associated with the $13 \mathrm{a}^{\prime}\left(\sigma_{\mathrm{Cl} L \mathrm{LP}}\right)$ orbital is evident in the experimental and theoretical results. However, its influence on the second nominally chlorine lone-pair orbital $\left(2 \mathrm{a}^{\prime \prime}\left(\pi_{\mathrm{Cl}} \mathrm{LP}\right)\right)$ appears negligible. A plausible explanation for these findings is that the $2 \mathrm{a}^{\prime \prime}\left(\pi_{\mathrm{Cl}}\right.$ LP) orbital, which is orientated perpendicular to the molecular plane, interacts with the $\pi$ orbitals in the ring system and hence its atomic character is reduced. 
This manuscript was accepted by J. Chem. Phys. Click here to see the version of record. Version 20

\section{SUPPLEMENTARY MATERIAL}

See supplementary material for the PT2 relaxation energy for the highest occupied orbitals of 2-chloropyridine and 3-chloropyridine, and for the experimental and theoretical photoelectron anisotropy parameters and branching ratios of 3-chloropyridine.

\section{ACKNOWLEDGMENTS}

A.B.T. gratefully acknowledges the grant, Grant No. 4.1671.2017/4.6, from the Ministry of Education and Science of the Russian Federation. The authors are indebted to D. Yu. Soshnikov for help with the electronic structure calculations. D.M.P.H. is grateful to the Science and Technology facilities Council (United Kingdom) for financial support. 
This manuscript was accepted by J. Chem. Phys. Click here to see the version of record. Version 20

\section{REFERENCES}

[1] A.B. Trofimov, D.M.P. Holland, I. Powis, R.C. Menzies, A.W. Potts, L. Karlsson, E.V.Gromov, I.L. Badsyuk, and J. Schirmer, J. Chem. Phys.146, 244307 (2017).

[2] I. Reineck, R. Maripuu, H. Veenhuizen, L. Karlsson, K. Siegbahn, M.S. Powar, W.N. $\mathrm{Zu}$, J.M. Rong, and S.H. Al-Shamma, J. Electron Spectrosc. Relat. Phenom. 27, 15 (1982).

[3] N. Kishimoto and K. Ohno, J. Phys. Chem. A 104, 6940 (2000).

[4] S.Y. Lui, K. Alnama, J. Matsumoto, K. Nishizawa, H. Kohguchi, Y.P. Lee, and T. Suzuki, J. Phys. Chem. A 115, 2953 (2011).

[5] J. Schirmer, L.S. Cederbaum, and O. Walter, Phys. Rev. A 28, 1237 (1983).

[6] J. Schirmer and G. Angonoa, J. Chem. Phys. 91, 1754 (1989).

[7] W. von Niessen, J. Schirmer, and L.S. Cederbaum, Comput. Phys. Rep. 1, 57 (1984).

[8] V.G. Zakrzewski and J.V. Ortiz, Int. J. Quantum Chem. Suppl. 28, 23 (1994).

[9] J.V. Ortiz, in Computational Chemistry: Reviews of Current Trends, edited by J. Leszcynski (World Scientific, Singapore, 1997), Vol. 2, p.1.

[10] S.T. Manson, D.J. Kennedy, A.F. Starace, and D. Dill, Planet. Space Sci. 22, 1535 (1974).

[11] J.W. Cooper, Phys. Rev. 128, 681 (1962).

[12] U. Fano and J.W. Cooper, Rev. Mod. Phys. 2, 177 (1966).

[13] S.T. Manson, A. Msezane, A.F. Starace, and S. Shahabi, Phys. Rev. A 20, 1005 (1979).

[14] Y. Hikosaka, J.H.D. Eland, T.M. Watson, and I. Powis, J. Chem. Phys. 115, 4593 (2001).

[15] D.W. Turner, C. Baker, A.D. Baker, and C.R. Brundle, Molecular Photoelectron Spectroscopy, (Wiley-Interscience, London, 1970).

[16] A. Modelli and G. Distefano, J. Electron Spectrosc. Relat. Phenom. 23, 323 (1981).

[17] G.R. Caine, S.J. Dunne, and E.I. von Nagy-Felsobuki, J. Heterocyclic Chem. 32, 89 (1995).

[18] C. Ma, L. Yao, and M. Ge, J. Mol. Struct. 881, 123 (2008). 
[19] M.R. Basila and D.J. Clancy, J. Phys. Chem. 67, 1551 (1963).

[20] D. Stefanović and H.F. Grützmacher, Org. Mass Spectrom. 9, 1052 (1974).

[21] L.S. Cederbaum, W. Domcke, J. Schirmer, and W. von Niessen, Adv. Chem. Phys. 65, 115 (1986).

[22] D.M.P. Holland, Phys. Scripta 36, 22 (1987).

[23] P. Finetti, D.M.P. Holland, C.J. Latimer, C. Binns, F.M. Quinn, M.A. Bowler, A.F. Grant, and C.S. Mythen, Nucl. Instrum. Methods Phys. Res. B 184, 627 (2001).

[24] D.M.P. Holland, M.A. MacDonald, M.A. Hayes, P. Baltzer, L. Karlsson, M. Lundqvist, B. Wannberg, and W. von Niessen, Chem. Phys. 188, 317 (1994).

[25] S.H. Southworth, A.C. Parr, J.E. Hardis, J.E. Dehmer, and D.M.P. Holland, Nucl. Instrum. Methods Phys. Res. A 246, 782 (1986).

[26] A.B. Trofimov, J. Schirmer, D.M.P. Holland, L. Karlsson, R. Maripuu, K. Siegbahn, and A.W. Potts, Chem. Phys. 263, 167 (2001).

[27] A.W. Potts, A.B. Trofimov, J. Schirmer, D.M.P. Holland, and L. Karlsson, Chem. Phys. 271, 337 (2001).

[28] A.B. Trofimov, J. Schirmer, D.M.P. Holland, A.W. Potts, L. Karlsson, R. Maripuu, and K. Siegbahn, J. Phys. B: At., Mol. Opt. Phys. 35, 5051 (2002).

[29] D.M.P. Holland, A.W. Potts, L. Karlsson, I.L. Zaytseva, A.B. Trofimov, and J. Schirmer, Chem. Phys. 352, 205 (2008).

[30] A.W.Potts, D.M.P. Holland, I. Powis, L. Karlsson, A.B.Trofimov, and I.L. Bodzuk, Chem. Phys. 415, 84 (2013).

[31] I. Powis, A.B. Trofimov, I.L. Bodzuk, D.M.P. Holland, A.W. Potts, and L. Karlsson, Chem.Phys. 415, 291 (2013).

[32] D.M.P. Holland, I. Powis, A.B. Trofimov, I.L. Bodzuk, D. Yu. Soshnikov, A.W. Potts, and L. Karlsson, Chem. Phys. 448, 61 (2015).

[33] The ADC(3) code originally written by G. Angonoa, O. Walter, and J. Schirmer; Further developed by M.K. Scheller and A.B. Trofimov. 
This manuscript was accepted by J. Chem. Phys. Click here to see the version of record. Version 20

[34] M.W. Schmidt, K.K. Baldridge, J.A. Boatz, S.T. Elbert, M.S. Gordon, J.H. Jensen, S. Koseki, N. Matsunaga, K.A. Nguyen, S. Su, T.L. Windus, M. Dupuis, and J.A. Montgomery, J. Comp. Chem. 14, 1347 (1993).

[35] M.S. Gordon, M.W. Schmidt, in Advances in Electronic Structure Theory: GAMESS a decade later, edited by C. E. Dykstra, G. Frenking, K. S. Kim, and G. E. Scuseria (Elsevier, Amsterdam, 2005), p. 1167.

[36] T.H. Dunning, J. Chem. Phys. 90, 1007 (1989).

[37] R.A. Kendall, T.H. Dunning, and R.J. Harrison, J. Chem. Phys. 96, 6769 (1992).

[38] H. Sekino and R.J. Bartlett, Int. J. Quantum Chem. Suppl. 18, 255 (1984).

[39] A.I. Krylov, Annu. Rev. Phys. Chem. 59, 433 (2008).

[40] M. Nooijen and J.G. Snijders, Int. J. Quantum Chem. Suppl. 26, 55 (1992).

[41] J.F. Stanton and J. Gauss, J. Chem. Phys. 103, 1064 (1995).

[42] Y. Shao et al., Mol. Phys. 113, 184 (2015).

[43] J. F. Stanton and J. Gauss, J. Chem. Phys. 111, 8785 (1999).

[44] CFOUR, Coupled cluster techniques for Computational Chemistry, a quantumchemical program package by J. F. Stanton, J. Gauss, M. E. Harding, and P. G. Szalay, with contributions from A. A. Auer, R. J. Bartlett, U. Benedikt, C. Berger, D. E. Bernholdt, Y. J. Bomble, L. Cheng, O. Christiansen, M. Heckert, O. Heun, C. Huber, T.-C. Jagau, D. Jonsson, J. Jusélius, K. Klein, W. J. Lauderdale, D. A. Matthews, T. Metzroth, L. A. Mück, D. P. O’Neill, D. R. Price, E. Prochnow, C. Puzzarini, K. Ruud, F. Schiffmann, W. Schwalbach, C. Simmons, S. Stopkowicz, A. Tajti, J. Vázquez, F. Wang, and J. D. Watts and the integral packages MOLECULE (J. Almlöf and P. R. Taylor), PROPS (P. R. Taylor), ABACUS (T. Helgaker, H. J. Aa. Jensen, P. Jørgensen, and J. Olsen), and ECP routines by A. V. Mitin and C. van Wüllen, for the current version, see http://www.cfour.de.

[45] M.J. Frisch et al., Gaussian 09, Revision C.01, Gaussian Inc. Wallingford, CT, 2010.

[46] R. Dennington, T. Keith, and J. Millam, GaussView, Versiion 5 Semichem Inc., Shawnee Mission, KS, 2009. 
This manuscript was accepted by J. Chem. Phys. Click here to see the version of record. Version 20

[47] G. Schaftenaar and J.H. Noordik, "Molden: A pre- and post-processing program for molecular and electronic structures", J. Comput.-Aided Mol. Des 14, 123 (2000).

[48] J. Schirmer, M. Braunstein, M.-T. Lee, and V. McKoy, in VUV and Soft X-Ray Photoionization, edited by U. Becker and D. A. Shirley (Plenum Press, New York, 2001), p. 105.

[49] G. Born, H.A. Kurtz, and Y. Öhrn, J. Chem. Phys. 68, 74 (1978).

[50] D.M.P. Holland, I. Powis, G. Öhrwall, L. Karlsson, W. von Niessen, Chem. Phys. 326, 535 (2006).

[51] J.G. Norman, J. Chem. Phys. 61, 4630 (1974).

[52] R.S. Mulliken, J. Chem. Phys. 23, 1833 (1955).

[53] D. Feller, J. Chem. Phys. 96, 6104 (1992).

[54] D.M.P. Holland, L. Karlsson, and W. von Niessen, J. Electron Spectrosc. Relat. Phenom. 113, 221 (2001).

[55] J.P. Foster and F. Weinhold, J.Am. Chem. Soc., 102, 7211 (1980).

[56] F. Weinhold and C. R. Landis, Discovering Chemistry with Natural Bond Orbitals (Wiley-VCH, Hoboken, 2012).

[57] E.D. Glendening, J.K. Badenhoop, A.E. Reed, J.E. Carpenter, J.A. Bohmann, C.M. Morales, C.R. Landis, and F. Weinhold NBO 6.0, Theoretical Chemistry Institute, University of Wisconsin, Madison.

[58] E. D. Glendening, C. R. Landis, and F. Weinhold, J. Comput. Chem. 34, 1429 (2013).

[59] I. Powis, D.M.P. Holland, E. Antonsson, M. Patanen, C. Nicolas, C. Miron, M. Schneider, D. Yu. Soshnikov, A Dreuw, and A.B. Trofimov, J. Chem. Phys. 143, 144304 (2015).

[60] A.W. Potts, D. Edvardsson, L. Karlsson, D.M.P. Holland, M.A. MacDonald, M.A. Hayes, R. Maripuu, K. Siegbahn, and W. von Niessen, Chem. Phys. 254, 385 (2000). 
Table I. Energy regions used in the analysis of the photoelectron spectra of 2-chloropyridine and 3chloropyridine.

\begin{tabular}{|c|c|c|c|}
\hline \multirow{2}{*}{ Region } & \multicolumn{2}{|c|}{ Binding energy range $(\mathrm{eV})$} & \\
\hline & 2-chloropyridine & 3-chloropyridine & \\
\hline 1 & $9.00-10.45$ & $8.80-10.30$ & $4 a^{\prime \prime}(\pi), 14 a^{\prime}\left(\sigma_{N L P}\right)$ \\
\hline 2 & $10.45-11.25$ & $10.30-11.35$ & $3 a^{\prime \prime}(\pi)$ \\
\hline 3 & $11.25-11.85$ & $11.35-12.17$ & $13 \mathrm{a}^{\prime}\left(\sigma_{\mathrm{Cl} L \mathrm{LP}}\right)$ \\
\hline 4 & $11.85-12.60$ & $12.17-12.80$ & $2 \mathrm{a}^{\prime \prime}\left(\pi_{\mathrm{Cl} \mathrm{LP}}\right)$ \\
\hline 5 & $12.60-13.45$ & $12.80-13.37$ & $12 \mathrm{a}^{\prime}(\sigma)$ \\
\hline 6 & $13.45-14.35$ & $13.37-14.32$ & $1 \mathrm{a}^{\prime \prime}(\pi), 11 \mathrm{a}^{\prime}(\sigma)$ \\
\hline 7 & $14.35-15.35$ & $14.32-15.30$ & $10 \mathrm{a}^{\prime}(\sigma)$ \\
\hline 8 & $15.35-15.95$ & $15.30-15.90$ & $9 \mathrm{a}^{\prime}(\sigma)$ \\
\hline 9 & $15.95-16.75$ & $15.90-16.85$ & $8 \mathrm{a}^{\prime}(\sigma)$ \\
\hline 10 & $16.75-18.50$ & 18.70 & $7 \mathrm{a}^{\prime}(\sigma)$ \\
\hline 11 & $18.50-21.50$ & $.70-21.50$ & $6 \mathrm{a}^{\prime}(\sigma), 5 \mathrm{a}^{\prime}(\sigma)$ \\
\hline 12 & $21.50-32.00$ & $21.50-32.00$ & $4 \mathrm{a}^{\prime}(\sigma), 3 \mathrm{a}^{\prime}(\sigma), 2 \mathrm{a}^{\prime}(\sigma), 1 \mathrm{a}^{\prime}(\sigma)$ \\
\hline
\end{tabular}


Table II. Mulliken atomic population in the outer valence molecular orbitals of 2-chloropyridine (units are electrons; sum over all atoms is 2) calculated at the HF/cc-pVDZ level.

\begin{tabular}{|c|c|c|c|c|c|c|c|c|c|c|c|c|c|}
\hline \multirow{2}{*}{ Atom } & $\begin{array}{c}4 \mathrm{a}^{\prime \prime} \\
(\pi)\end{array}$ & $\begin{array}{c}14 \mathrm{a}^{\prime} \\
\left(\sigma_{\mathrm{N} \mathrm{LP}}\right)\end{array}$ & $\begin{array}{c}3 \mathrm{a}^{\prime \prime} \\
(\pi)\end{array}$ & $\begin{array}{c}13 \mathrm{a}^{\prime} \\
\left(\sigma_{\mathrm{ClLP}}\right)\end{array}$ & $\begin{array}{c}2 \mathrm{a}^{\prime \prime} \\
\left(\pi_{\mathrm{Cl} \mathrm{LP}}\right)\end{array}$ & $\begin{array}{c}12 \mathrm{a}^{\prime} \\
(\sigma)\end{array}$ & $\begin{array}{c}1 \mathrm{a}^{\prime \prime} \\
(\pi)\end{array}$ & $\begin{array}{c}11 \mathrm{a}^{\prime} \\
(\sigma)\end{array}$ & $\begin{array}{c}10 \mathrm{a}^{\prime} \\
(\sigma)\end{array}$ & $\begin{array}{c}9 \mathrm{a}^{\prime} \\
(\sigma)\end{array}$ & $\begin{array}{c}8 \mathrm{a}^{\prime} \\
(\sigma)\end{array}$ & $\begin{array}{c}7 \mathrm{a}^{\prime} \\
(\sigma)\end{array}$ \\
\hline $\mathrm{N}$ & 0.02 & 1.22 & 0.60 & 0.03 & 0.05 & 0.14 & 0.48 & 0.32 & 0.03 & 0.04 & 0.30 & 0.16 \\
\hline $\mathrm{C}_{2}$ & 0.37 & 0.13 & 0.04 & 0.03 & 0.04 & 0.16 & 0.52 & 0.21 & 0.16 & 0.14 & 0.29 & 0.13 \\
$\mathrm{C}_{3}$ & 0.42 & 0.11 & 0.38 & 0.03 & 0.01 & 0.23 & 0.22 & 0.08 & 0.25 & 0.35 & 0.32 & 0.23 \\
\hline $\mathrm{C}_{4}$ & 0.02 & 0.03 & 0.63 & 0.02 & 0.12 & 0.26 & 0.14 & 0.49 & 0.12 & 0.08 & 0.33 & 0.27 \\
\hline $\mathrm{C}_{5}$ & 0.52 & 0.13 & 0.16 & 0.00 & 0.23 & 0.23 & 0.14 & 0.17 & 0.34 & 0.15 & 0.35 & 0.30 \\
\hline $\mathrm{C}_{6}$ & 0.35 & 0.15 & 0.14 & 0.01 & 0.18 & 0.18 & 0.24 & 0.07 & 0.29 & 0.27 & 0.31 & 0.23 \\
\hline $\mathrm{Cl}$ & 0.29 & 0.09 & 0.04 & 1.88 & 1.36 & 0.36 & 0.26 & 0.24 & 0.27 & 0.18 & 0.06 & 0.14 \\
\hline \hline
\end{tabular}


Table III. Mulliken atomic population in the outer valence molecular orbitals of 3-chloropyridine (units are electrons; sum over all atoms is 2) calculated at the HF/cc-pVDZ level.

\begin{tabular}{|c|c|c|c|c|c|c|c|c|c|c|c|c|}
\hline \multirow{2}{*}{ Atom } & $\begin{array}{c}4 \mathrm{a}^{\prime \prime} \\
(\pi)\end{array}$ & $\begin{array}{c}14 \mathrm{a}^{\prime} \\
\left(\sigma_{\mathrm{N} \mathrm{LP}}\right)\end{array}$ & $\begin{array}{c}3 \mathrm{a}^{\prime \prime} \\
(\pi)\end{array}$ & $\begin{array}{c}13 \mathrm{a}^{\prime} \\
\left(\sigma_{\mathrm{Cl} \mathrm{LP}}\right)\end{array}$ & $\begin{array}{c}2 \mathrm{a}^{\prime \prime} \\
\left(\pi_{\mathrm{Cl} \mathrm{LP}}\right)\end{array}$ & $\begin{array}{c}12 \mathrm{a}^{\prime} \\
(\sigma)\end{array}$ & $\begin{array}{c}1 \mathrm{a}^{\prime \prime} \\
(\pi)\end{array}$ & $\begin{array}{c}11 \mathrm{a}^{\prime} \\
(\sigma)\end{array}$ & $\begin{array}{c}10 \mathrm{a}^{\prime} \\
(\sigma)\end{array}$ & $\begin{array}{c}9 \mathrm{a}^{\prime} \\
(\sigma)\end{array}$ & $\begin{array}{c}8 \mathrm{a}^{\prime} \\
(\sigma)\end{array}$ & $\begin{array}{c}7 \mathrm{a}^{\prime} \\
(\sigma)\end{array}$ \\
\hline $\mathrm{N}$ & 0.02 & 1.13 & 0.48 & 0.14 & 0.28 & 0.17 & 0.34 & 0.30 & 0.05 & 0.05 & 0.35 & 0.12 \\
\hline $\mathrm{C}_{2}$ & 0.38 & 0.15 & 0.18 & 0.01 & 0.04 & 0.18 & 0.33 & 0.09 & 0.32 & 0.16 & 0.26 & 0.28 \\
\hline $\mathrm{C}_{3}$ & 0.49 & 0.11 & 0.09 & 0.03 & 0.08 & 0.19 & 0.43 & 0.11 & 0.31 & 0.18 & 0.35 & 0.09 \\
$\mathrm{C}_{4}$ & 0.04 & 0.03 & 0.66 & 0.05 & 0.00 & 0.22 & 0.22 & 0.58 & 0.02 & 0.07 & 0.30 & 0.31 \\
$\mathrm{C}_{5}$ & 0.30 & 0.10 & 0.47 & 0.05 & 0.08 & 0.23 & 0.17 & 0.15 & 0.13 & 0.49 & 0.34 & 0.14 \\
\hline $\mathrm{C}_{6}$ & 0.46 & 0.13 & 0.02 & 0.03 & 0.24 & 0.19 & 0.21 & 0.21 & 0.27 & 0.07 & 0.32 & 0.34 \\
\hline $\mathrm{Cl}$ & 0.31 & 0.22 & 0.10 & 1.67 & 1.27 & 0.37 & 0.28 & 0.01 & 0.49 & 0.33 & 0.06 & 0.06 \\
\hline \hline
\end{tabular}


Table IV. Energies $E(\mathrm{eV})$ and intensities $P$ of the outer valence vertical ionization transitions in 2-chloropyridine computed using the HF, OVGF and ADC(3) methods and the cc-pVDZ (DZ) basis set; vertical ionization energies $(\mathrm{eV})$ obtained using the EOM-IP-CCSD (CCSD) method in combination with the aug-cc-pVTZ (ATZ) basis set, and the continuum orbital version of EOM-EE-CC3 (CC3) method in combination with the cc-pVTZ (TZ) basis set. The $\mathrm{CC} 3$ vertical ionization energies extrapolated to the complete basis set limit $\left(\mathrm{CC} 3_{\infty}\right)$ and the experimental values (Exp.) are also listed.

\begin{tabular}{|c|c|c|c|c|c|c|c|c|c|c|}
\hline \multirow{2}{*}{$\begin{array}{c}\text { Molecular } \\
\text { orbital }\end{array}$} & \multirow{2}{*}{ Гуре } & \multicolumn{3}{|c|}{$\mathrm{HF} / \mathrm{DZ} \sim \mathrm{OVGF} / \mathrm{DZ}$} & \multicolumn{2}{|c|}{$\mathrm{ADC}(3) / \mathrm{DZ}$} & \multirow{2}{*}{$\begin{array}{c}\mathrm{CCSD} / \\
\mathrm{ATZ}\end{array}$} & \multirow{2}{*}{$\begin{array}{c}\mathrm{CC} 3 / \\
\mathrm{TZ} \\
\end{array}$} & \multirow{2}{*}{$\mathrm{CC} 3_{\infty}{ }^{\mathrm{a}}$} & \multirow{2}{*}{ Exp. } \\
\hline & & & $E$ & $P$ & $E$ & $P$ & & & & \\
\hline $4 a^{\prime \prime}$ & & 9.48 & 9.28 & 0.90 & 9.34 & 0.89 & 9.63 & 9.50 & 9.64 & 9.63 \\
\hline $14 \mathrm{a}^{\prime}$ & & 11.89 & 10.05 & 0.89 & 10.34 & 0.88 & 10.25 & 10.01 & 10.17 & 10.11 \\
\hline & $\pi$ & 10.79 & 10.32 & 0.89 & 10.53 & 0.88 & 10.79 & 10.64 & 10.77 & 10.75 \\
\hline & $\sigma_{\mathrm{Cl}}$ & 12.42 & 11.33 & 0.91 & 11.33 & 0.90 & 11.64 & 11.38 & 11.49 & 11.56 \\
\hline & 4 & 12.95 & 11.92 & 0.89 & 11.96 & 0.86 & 12.30 & 11.99 & 12.07 & 12.11 \\
\hline & $\sigma$ & 14.53 & 13.09 & 0.90 & 13.31 & 0.90 & 13.37 & 13.13 & 13.19 & 13.03 \\
\hline & $\pi$ & 15.70 & 14.07 & 0.83 & 14.09 & 0.56 & 14.49 & 13.85 & 13.88 & 13.91 \\
\hline $11 a^{\prime}$ & $\sigma$ & 16.01 & 14.22 & 0.89 & 14.44 & 0.88 & 14.45 & 14.10 & 14.14 & 13.98 \\
\hline $10 \mathrm{a}^{\prime}$ & $\sigma$ & 16.40 & 14.78 & 0.89 & 15.01 & 0.88 & 15.05 & 14.70 & 14.85 & 14.79 \\
\hline $9 \mathrm{a}^{\prime}$ & $\sigma$ & 17.92 & 16.21 & 0.88 & 16.28 & 0.85 & 16.36 & 15.96 & & 15.83 \\
\hline $8 a^{\prime}$ & $\sigma$ & 18.65 & 16.39 & 0.87 & 16.77 & 0.80 & 16.64 & & & 16.23 \\
\hline $7 \mathrm{a}^{\prime}$ & $\sigma$ & 19.60 & 17.59 & 0.86 & 17.69 & 0.73 & 17.76 & & & 17.23 \\
\hline
\end{tabular}

${ }^{a}$ The CC3 ionization energies for the complete basis set were estimated by adding the difference between the results for the cc-pVœZ and the cc-pVTZ basis sets (Table V) to the CC3/cc-pVTZ result.

${ }^{\mathrm{b}}$ Peak maxima, as obtained from the present photoelectron spectrum. 
Table V. Vertical ionization energies of 2-chloropyridine $(\mathrm{eV})$ calculated at the OVGF level of theory using a series of basis sets cc-pVnZ and aug-cc-pVnZ with improving quality $(n=\mathrm{D}, \mathrm{T}, \mathrm{Q}, 5)$ and results for the CBS limits $(n \rightarrow \infty)$.

\begin{tabular}{|c|c|c|c|c|c|c|c|c|c|}
\hline Basis set & $\begin{array}{c}4 \mathrm{a}^{\prime \prime} \\
(\pi)\end{array}$ & $\begin{array}{c}14 \mathrm{a}^{\prime} \\
\left(\sigma_{\mathrm{NLP}}\right)\end{array}$ & $\begin{array}{c}3 \mathrm{a}^{\prime \prime} \\
(\pi)\end{array}$ & $\begin{array}{c}13 \mathrm{a}^{\prime} \\
\left(\sigma_{\mathrm{Cl} \mathrm{LP}}\right)\end{array}$ & $\begin{array}{c}2 \mathrm{a}^{\prime \prime} \\
\left(\pi_{\mathrm{Cl} \mathrm{LP}}\right)\end{array}$ & $\begin{array}{c}12 \mathrm{a}^{\prime} \\
(\sigma)\end{array}$ & $\begin{array}{c}1 \mathrm{a}^{\prime \prime} \\
(\pi)\end{array}$ & $\begin{array}{c}11 \mathrm{a}^{\prime} \\
(\sigma)\end{array}$ & $\begin{array}{c}10 \mathrm{a}^{\prime} \\
(\sigma)\end{array}$ \\
\hline cc-pVDZ & 9.29 & 10.06 & 10.34 & 11.34 & 11.92 & 13.11 & 14.08 & 14.23 & 14.79 \\
\hline aug-cc-pVDZ & 9.46 & 10.33 & 10.55 & 11.60 & 12.16 & 13.32 & 14.30 & 14.43 & 14.98 \\
\hline cc-pVTZ & 9.48 & 10.26 & 10.63 & 11.59 & 12.13 & 13.25 & 14.20 & 14.36 & 14.90 \\
\hline aug-cc-pVTZ & 9.55 & 10.36 & 10.71 & 11.70 & 12.23 & 13.33 & 14.29 & 14.44 & 14.98 \\
\hline cc-pVQZ & 9.56 & 10.34 & 10.71 & 11.73 & 12.24 & 13.32 & 14.27 & 14.43 & 14.97 \\
\hline aug-cc-pVQZ & 9.59 & 10.38 & 10.75 & 11.77 & 12.28 & 13.35 & 14.31 & 14.46 & 15.00 \\
\hline cc-pV5Z & 9.59 & 10.37 & 10.74 & 11.76 & 12.27 & 13.35 & 14.29 & 14.45 & 15.00 \\
\hline cc-pVoOZ & 9.61 & 10.39 & 10.75 & 11.81 & 12.31 & 13.38 & 14.32 & 14.48 & 15.05 \\
\hline \hline
\end{tabular}


Table VI. HF IPs $(\mathrm{eV})$ and relaxation energies, $\Delta E^{R}(\mathrm{eV})$, with respect to the HF results for the highest occupied orbitals of 2-chloropyridine and 3-chloropyridine, computed using the $\triangle \mathrm{SCF}$ method and PT2 approach. $\Delta E_{\pi}^{R}(\mathrm{eV})$ is the contribution of the $\pi$-orbitals to the total relaxation energy. The percent of $\Delta E_{\pi}^{R}$ in the total $\Delta E^{R}$ is shown in parentheses. Calculations performed using the $6-31 \mathrm{G}^{*}$ basis set.

\begin{tabular}{|c|c|c|c|c|c|}
\hline $\begin{array}{l}\text { Molecule and } \\
\text { quantity }\end{array}$ & $\begin{array}{l}4 \mathrm{a}^{\prime \prime} \\
(\pi)\end{array}$ & $\begin{array}{c}14 \mathrm{a}^{\prime} \\
\left(\sigma_{\mathrm{N} \mathrm{LP}}\right)\end{array}$ & $\begin{array}{l}3 \mathrm{a}^{\prime \prime} \\
(\pi)\end{array}$ & & $\begin{array}{c}2 \mathrm{a}^{\prime \prime} \\
\left(\pi_{\mathrm{Cl} L \mathrm{LP}}\right)\end{array}$ \\
\hline \multicolumn{6}{|l|}{ 2-chloropyridine } \\
\hline IP (HF) & 9.46 & 11.86 & 10.75 & & 12.92 \\
\hline$\Delta E^{R}(\mathrm{PT} 2)$ & -0.88 & -2.62 & & -1.88 & -0.95 \\
\hline$\Delta E_{\pi}^{R}(\mathrm{PT} 2)$ & $\begin{array}{c}-0.12 \\
(14)\end{array}$ & $\begin{array}{c}-1.36 \\
(52)\end{array}$ & & $\begin{array}{l}-0.89 \\
(47)\end{array}$ & $\begin{array}{l}-0.19 \\
(20)\end{array}$ \\
\hline$\Delta E^{R}(\Delta \mathrm{SCF})^{\mathrm{a}}$ & -0.82 & -2.84 & & & \\
\hline \multicolumn{6}{|l|}{ 3-chloropyridine } \\
\hline IP (HF) & 9.43 & 11.60 & 10.61 & 12.80 & 13.39 \\
\hline$\Delta E^{R}(\mathrm{PT} 2)$ & -0.90 & -2.19 & -1.09 & -1.44 & -0.91 \\
\hline$\Delta E_{\pi}^{R}(\mathrm{PT} 2)$ & $\begin{array}{l}-0.13 \\
(15)\end{array}$ & & $\begin{array}{r}-0.13 \\
(12)\end{array}$ & $\begin{array}{l}-0.65 \\
(45)\end{array}$ & $\begin{array}{l}-0.19 \\
(21)\end{array}$ \\
\hline$\Delta E^{R}(\triangle \mathrm{SCF})^{\mathrm{a}}$ & -0.83 & -2.74 & & & \\
\hline
\end{tabular}

${ }^{a}$ The $\triangle \mathrm{SCF}$ results obtained using the cc-pVTZ basis set. The full ionization energies of the $4 \mathrm{a}^{\prime \prime}$ and $14 \mathrm{a}^{\prime}$ orbitals at the $\triangle \mathrm{SCF} / \mathrm{cc}-\mathrm{pVTZ}$ level are 8.68 and $9.10 \mathrm{eV}$ (2-chloropyridine), and 8.64 and $8.94 \mathrm{eV}$ (3-chloropyridine), respectively. 
Table VII. Energies $E(\mathrm{eV})$ and intensities $P$ of the outer valence vertical ionization transitions in 3chloropyridine computed using the HF, OVGF and ADC(3) methods and the cc-pVDZ (DZ) basis set; vertical ionization energies $(\mathrm{eV})$ obtained using the EOM-IP-CCSD (CCSD) method in combination with the aug-ccpVTZ (ATZ) basis set, and continuum orbital version of EOM-EE-CC3 (CC3) method in combination with the cc-pVTZ (TZ) basis set. The CC3 vertical ionization energies extrapolated to the complete basis set limit $\left(\mathrm{CC}_{\infty}\right)$ and the experimental values (Exp.) are also listed.

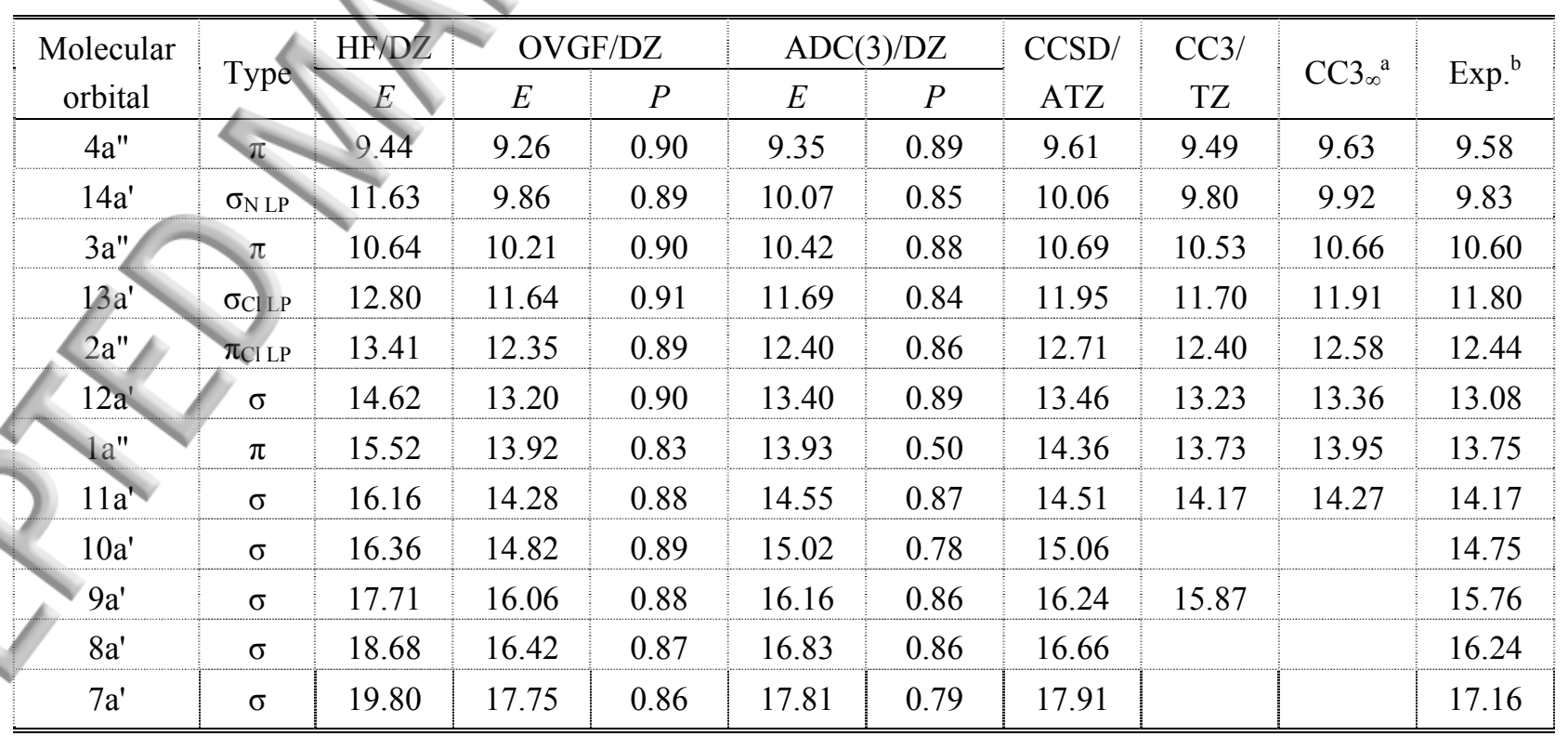

${ }^{a}$ The $\mathrm{CC} 3$ ionization energies for the complete basis set were estimated by adding the difference between the results for the cc-pVoZ and the cc-pVTZ basis sets (Table VIII) to the CC3/cc-pVTZ result.

${ }^{\mathrm{b}}$ Peak maxima, as obtained from the present photoelectron spectrum. 
Table VIII. Vertical ionization energies of 3-chloropyridine $(\mathrm{eV})$ calculated at the OVGF level of theory using a series of basis sets cc-pVnZ and aug-cc-pVnZ with improving quality $(n=\mathrm{D}, \mathrm{T}, \mathrm{Q}, 5)$ and results for the CBS limits $(n \rightarrow \infty)$.

\begin{tabular}{|c|c|c|c|c|c|c|c|c|c|}
\hline Basis set & $\begin{array}{c}4 \mathrm{a}^{\prime \prime} \\
(\pi)\end{array}$ & $\begin{array}{c}14 \mathrm{a}^{\prime} \\
\left(\sigma_{\mathrm{NLP}}\right)\end{array}$ & $\begin{array}{c}3 \mathrm{a}^{\prime \prime} \\
(\pi)\end{array}$ & $\begin{array}{c}13 \mathrm{a}^{\prime} \\
\left(\sigma_{\mathrm{Cl} L \mathrm{LP}}\right)\end{array}$ & $\begin{array}{c}2 \mathrm{a}^{\prime \prime} \\
\left(\pi_{\mathrm{Cl} \text { LP }}\right)\end{array}$ & $\begin{array}{c}12 \mathrm{a}^{\prime} \\
(\sigma)\end{array}$ & $\begin{array}{c}1 \mathrm{a}^{\prime \prime} \\
(\pi)\end{array}$ & $\begin{array}{c}11 \mathrm{a}^{\prime} \\
(\sigma)\end{array}$ & $\begin{array}{c}10 \mathrm{a}^{\prime} \\
(\sigma)\end{array}$ \\
\hline cc-pVDZ & 9.27 & 9.87 & 10.23 & 11.65 & 12.36 & 13.21 & 13.94 & 14.29 & 14.82 \\
\hline aug-cc-pVDZ & 9.44 & 10.14 & 10.43 & 11.91 & 12.61 & 13.42 & 14.14 & 14.48 & 15.04 \\
\hline cc-pVTZ & 9.46 & 10.09 & 10.52 & 11.88 & 12.56 & 13.36 & 14.02 & 14.42 & 14.94 \\
\hline aug-cc-pVTZ & 9.53 & 10.18 & 10.60 & 11.98 & 12.66 & 13.44 & 14.15 & 14.49 & 15.03 \\
\hline cc-pVQZ & 9.54 & 10.17 & 10.60 & 12.01 & 12.67 & 13.43 & 14.13 & 14.48 & 15.02 \\
\hline aug-cc-pVQZ & 9.57 & 10.21 & 10.63 & 12.05 & 12.71 & 13.46 & 14.16 & 14.51 & 15.05 \\
\hline cc-pV5Z & 9.57 & 10.20 & 10.63 & 12.04 & 12.70 & 13.46 & 14.15 & 14.50 & 15.05 \\
\hline cc-pVoOZ & 9.59 & 10.22 & 10.64 & 12.09 & 12.74 & 13.49 & 14.27 & 14.52 & 15.10 \\
\hline \hline
\end{tabular}




\section{Figure captions}

Figure 1. Schematic representation of pyridine, 2-chloropyridine and 3-chloropyridine, showing the atomic labelling. For 2-chloropyridine and 3-chloropyridine the molecular plane coincides with the xy coordinate plane. For pyridine the molecular plane coincides with the yz coordinate plane and the $\mathrm{z}$ axis is the principle $\mathrm{C}_{2 \mathrm{v}}$ point group axis.

Figure 2. A diagram correlating the outer valence molecular orbitals of pyridine, 2chloropyridine and 3-chloropyridine based on the vertical ionization energies computed using the EOM-IP-CCSDT/cc-pVTZ approach for the 7a1 ( $\left.\sigma_{\mathrm{N}} \mathrm{LP}\right)$, $1 \mathrm{a}_{2}(\pi), 2 \mathrm{~b}_{1}(\pi)$ and $5 \mathrm{~b}_{2}(\sigma)$ orbitals of pyridine, and the EOM-IP-CCSD/aug-ccpVTZ approach otherwise.

Figure 3. Plots of the five highest occupied molecular orbitals of 2-chloropyridine (left) and 3-chloropyridine (right) produced using the results of the HF/6-31G* calculations.

Figure 4. Contour plots of the total electron density differences showing the effects of orbital relaxation for the ${ }^{2} \mathrm{~A}^{\prime}\left(14 \mathrm{a}^{\prime-1}\right)$ and the ${ }^{2} \mathrm{~A}^{\prime \prime}\left(4 \mathrm{a}^{\prime \prime-1}\right)$ cationic states of 2chloropyridine. The differences showing the relaxation effect were obtained by subtracting the densities obtained from the ROHF/cc-pVTZ calculations for the cationic states from the density constructed using the ${ }^{1} \mathrm{~A}^{\prime}$ ground state $\mathrm{HF} / \mathrm{cc}-$ pVTZ orbitals. The red and blue contours indicate increase and decrease in electron density, respectively. The effect on the ionization energy is also shown. The heterocyclic rings are oriented to have the $\mathrm{N}$ atom at their lower vertex.

Figure 5. Plots of the virtual orbitals for 2-chloropyridine (left) and 3-chloropyridine (right) produced using the results of the HF/6-31G* calculations. The two lowest unoccupied $\pi$-orbitals and the $\mathrm{Cl} 4 \mathrm{p}$ Rydberg orbital play an important role in the relaxation accompanying ionization of the $\sigma_{\mathrm{N}} \mathrm{LP}$ and $\sigma_{\mathrm{Cl}} \mathrm{LP}$ lone-pairs. 
Figure 6. (a) The valence shell photoelectron spectrum of 2-chloropyridine recorded at a photon energy of $80 \mathrm{eV}$ and at $\theta=0^{\circ}$. (b) The theoretical photoelectron spectrum of 2-chloropyridine obtained using the $\mathrm{ADC}(3)$ method.

Figure 7. The outer valence shell photoelectron spectrum of 2-chloropyridine recorded at a photon energy of $42.5 \mathrm{eV}$ for $\theta=0^{\circ}$ (a) and $\theta=90^{\circ}(\mathrm{b})$.

Figure 8. (a) The valence shell photoelectron spectrum of 3-chloropyridine recorded at a photon energy of $80 \mathrm{eV}$ and at $\theta=0^{\circ}$. (b) The theoretical photoelectron spectrum of 3-chloropyridine obtained using the $\operatorname{ADC}(3)$ method.

Figure 9. The outer valence shell photoelectron spectrum of 3-chloropyridine recorded at a photon energy of $42.5 \mathrm{eV}$ for $\theta=0^{\circ}$ (a) and $\theta=90^{\circ}$ (b).

Figure 10. Contour plots of the total electron density differences showing the effects of orbital relaxation for the ${ }^{2} \mathrm{~A}^{\prime}\left(14 \mathrm{a}^{\prime-1}\right)$ and the ${ }^{2} \mathrm{~A}^{\prime \prime}\left(4 \mathrm{a}^{\prime \prime-1}\right)$ cationic states of 3chloropyridine. The differences showing the relaxation effect were obtained by subtracting the densities obtained from the ROHF/cc-pVTZ calculations for the cationic states from the density constructed using the ${ }^{1} \mathrm{~A}^{\prime}$ ground state $\mathrm{HF} / \mathrm{cc}-$ pVTZ orbitals. The red and blue contours indicate increase and decrease in electron density, respectively. The effect on the ionization energy is also shown. The heterocyclic rings are oriented to have the $\mathrm{N}$ atom at their lower vertex.

Figure 11. The experimental and theoretical (CMS - X $\alpha$ ) photoelectron anisotropy parameters of 2-chloropyridine associated with the binding energy regions $1-12$ specified in Table I.

Figure 12. The experimental and theoretical (CMS - X $\alpha$ ) photoelectron intensity branching ratios of 2-chloropyridine associated with the binding energy regions $1-10$ specified in Table I. 


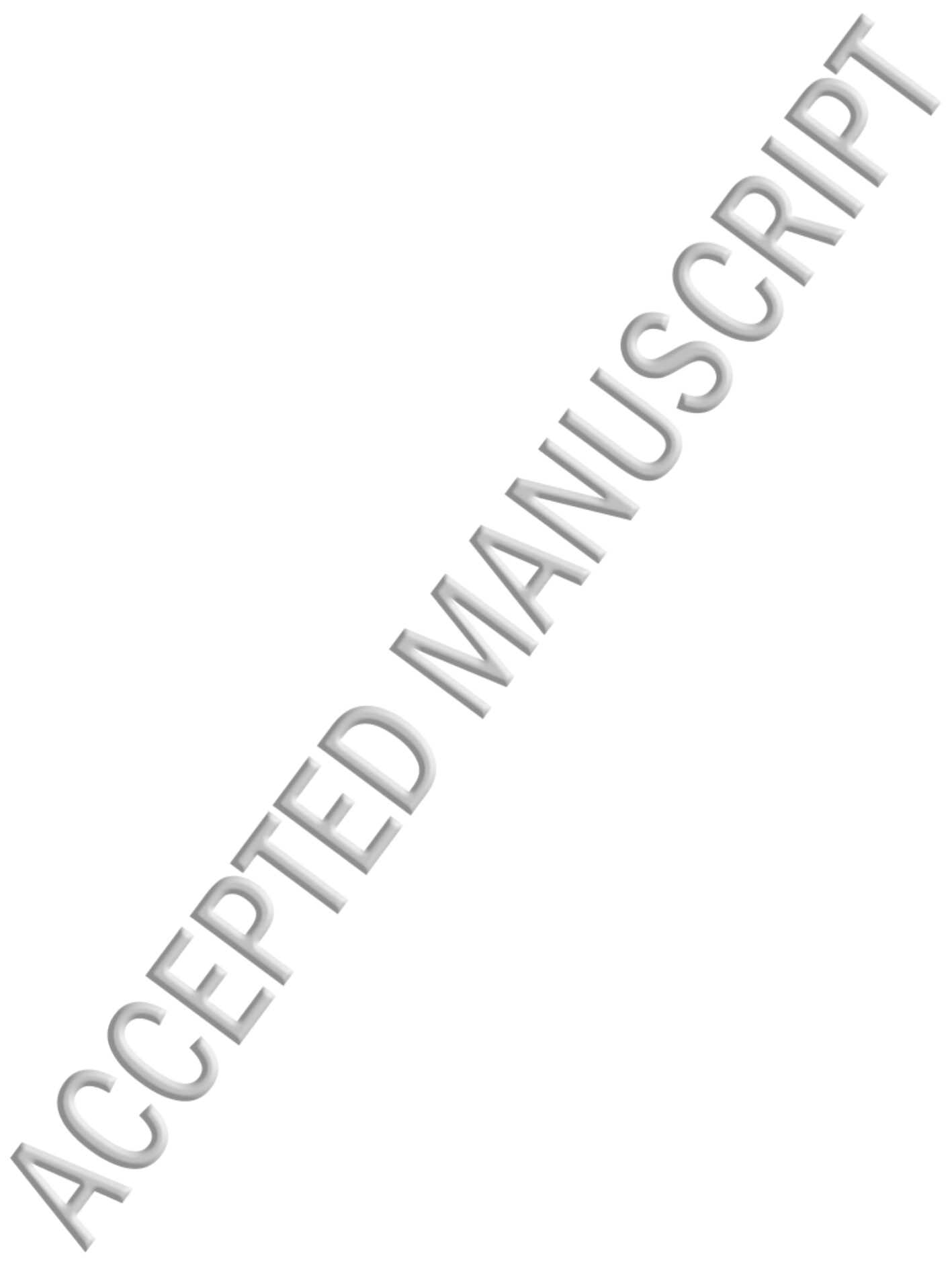



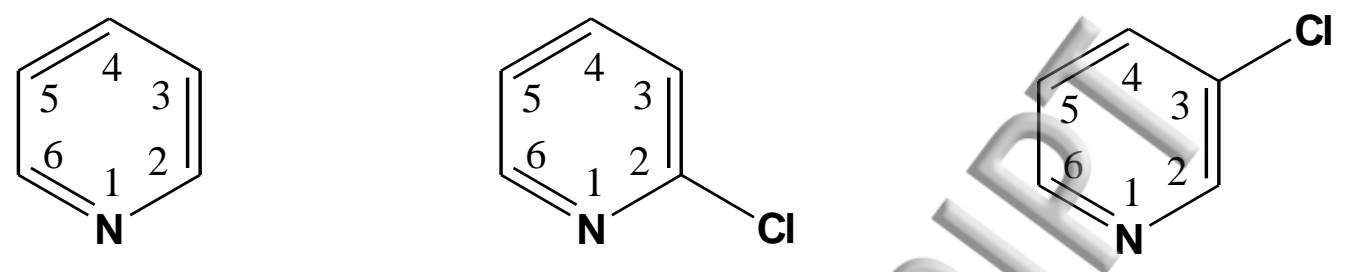

pyridine

2-chloropyridine

3-chloropyridine 


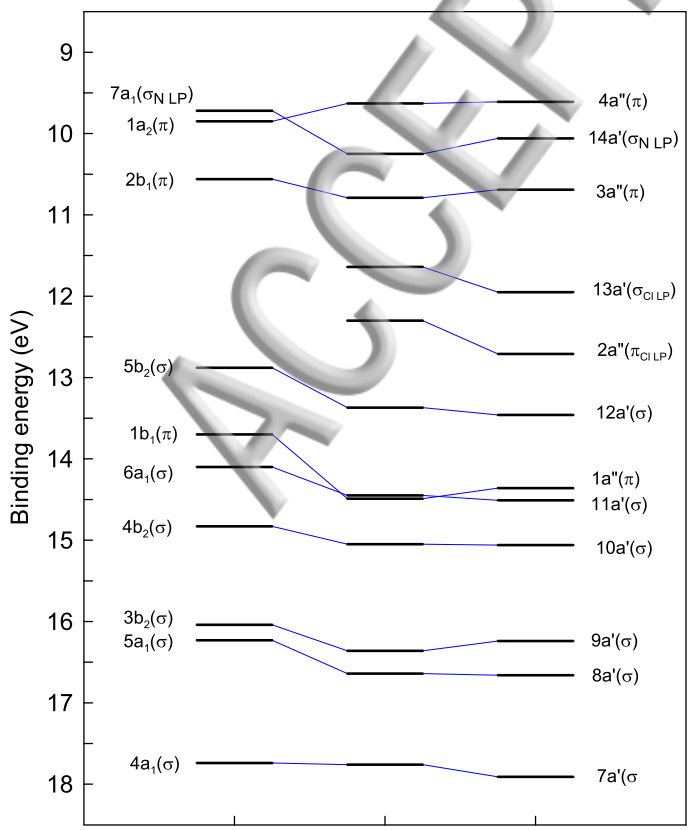

pyridine 2-chloropyridine 3-chloropyridine 


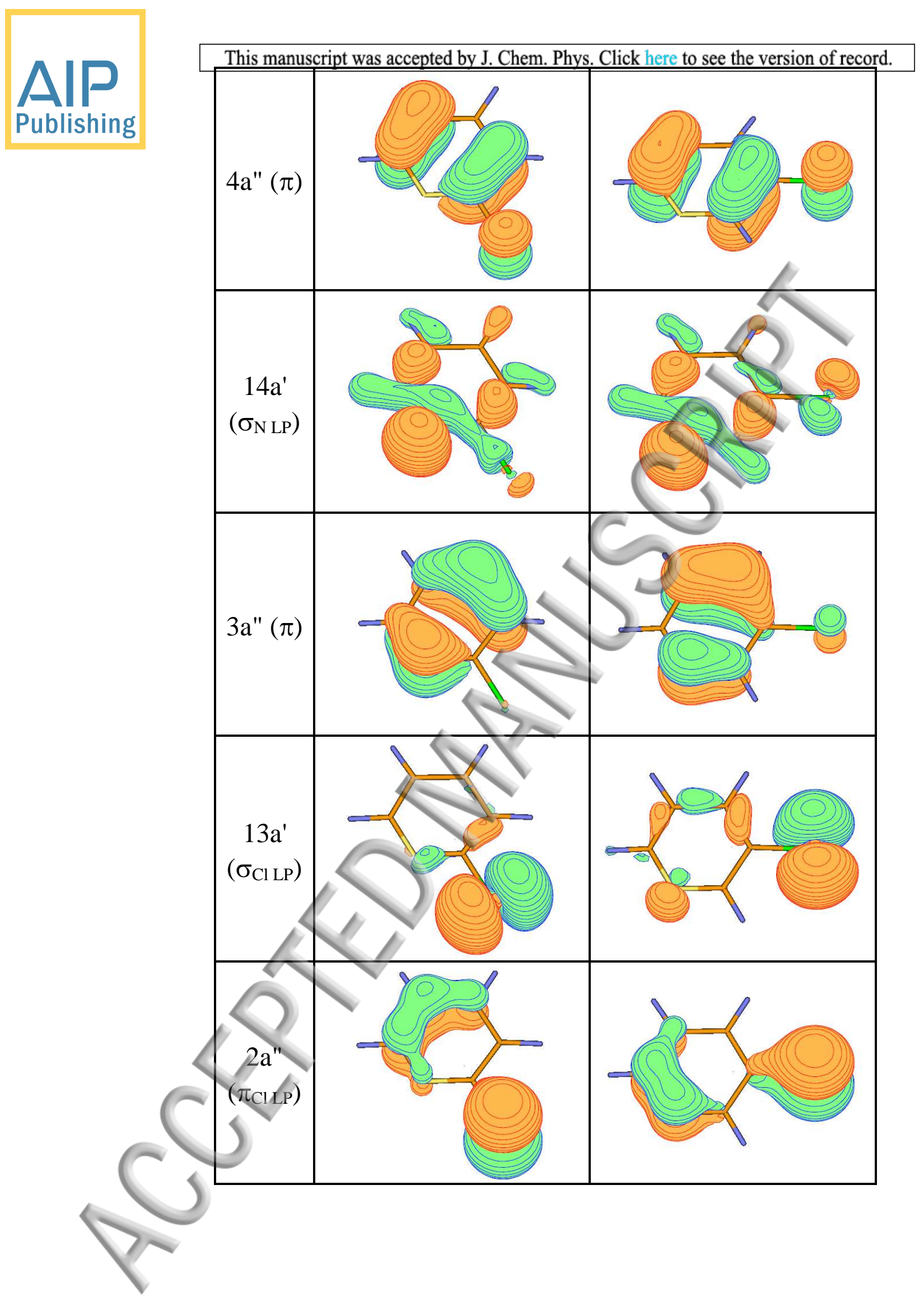




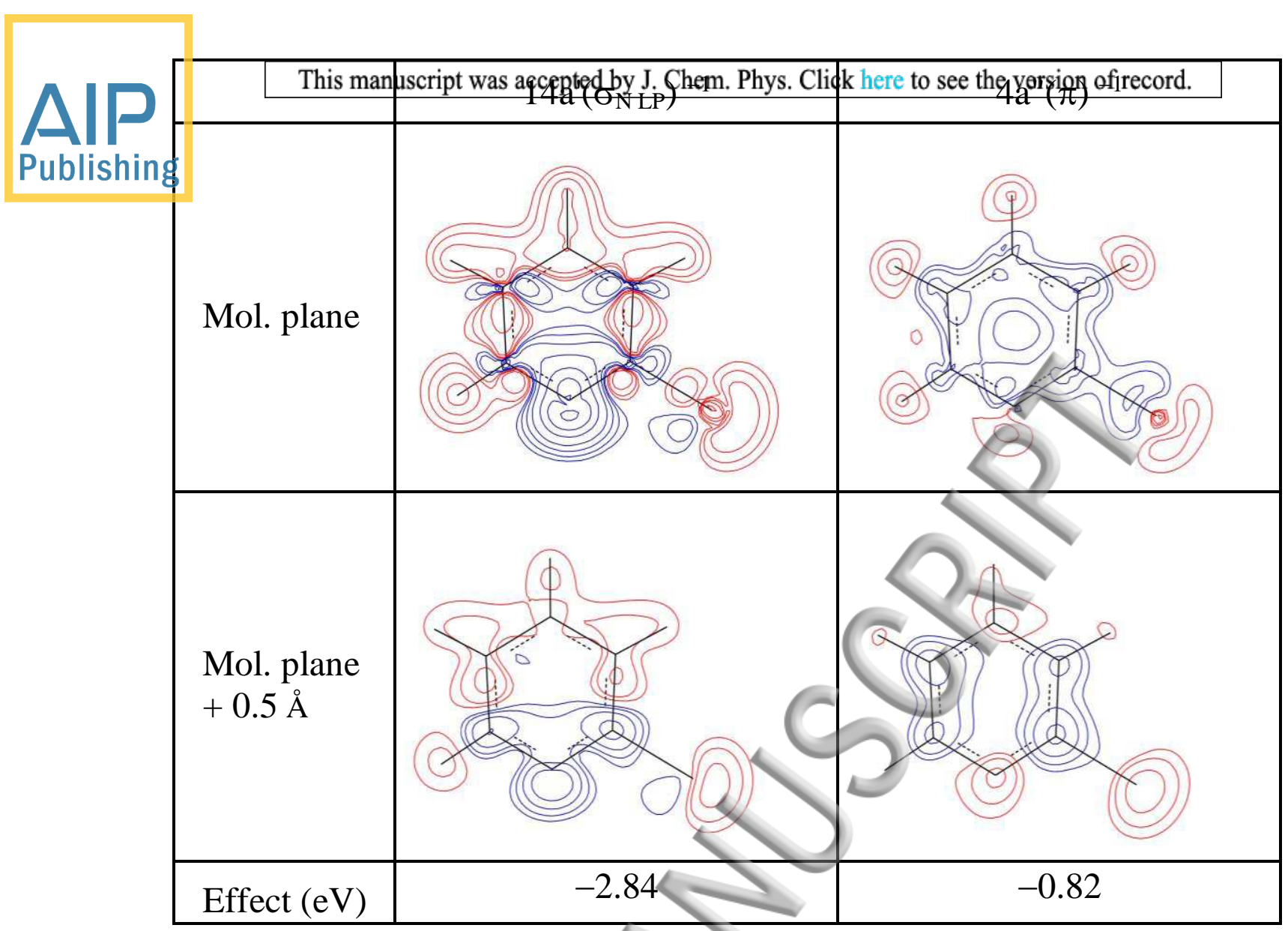




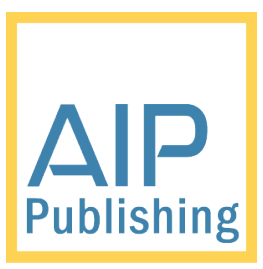

This manuscript was accepted by J. Chem. Phys. Click here to see the version of record.

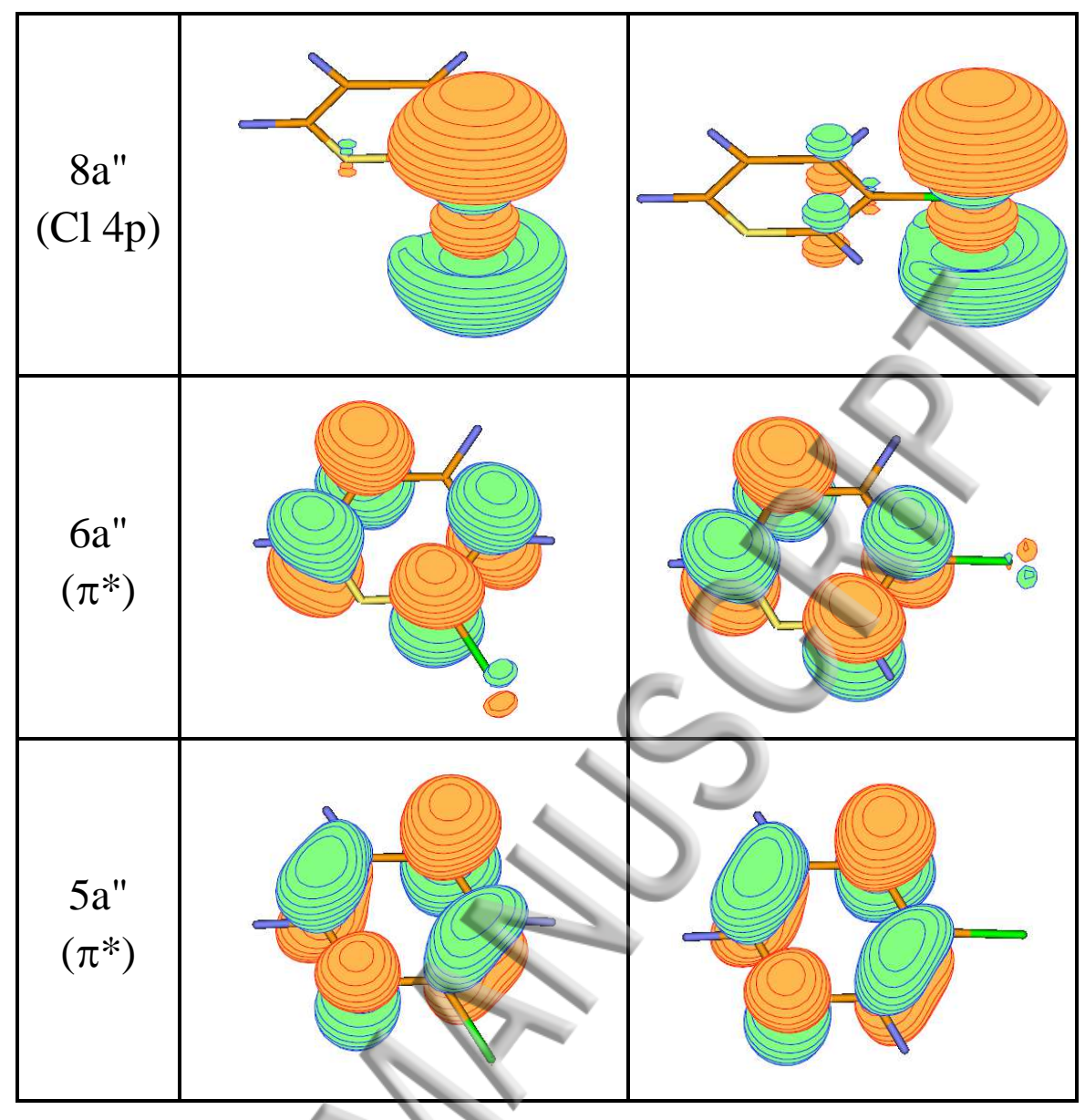




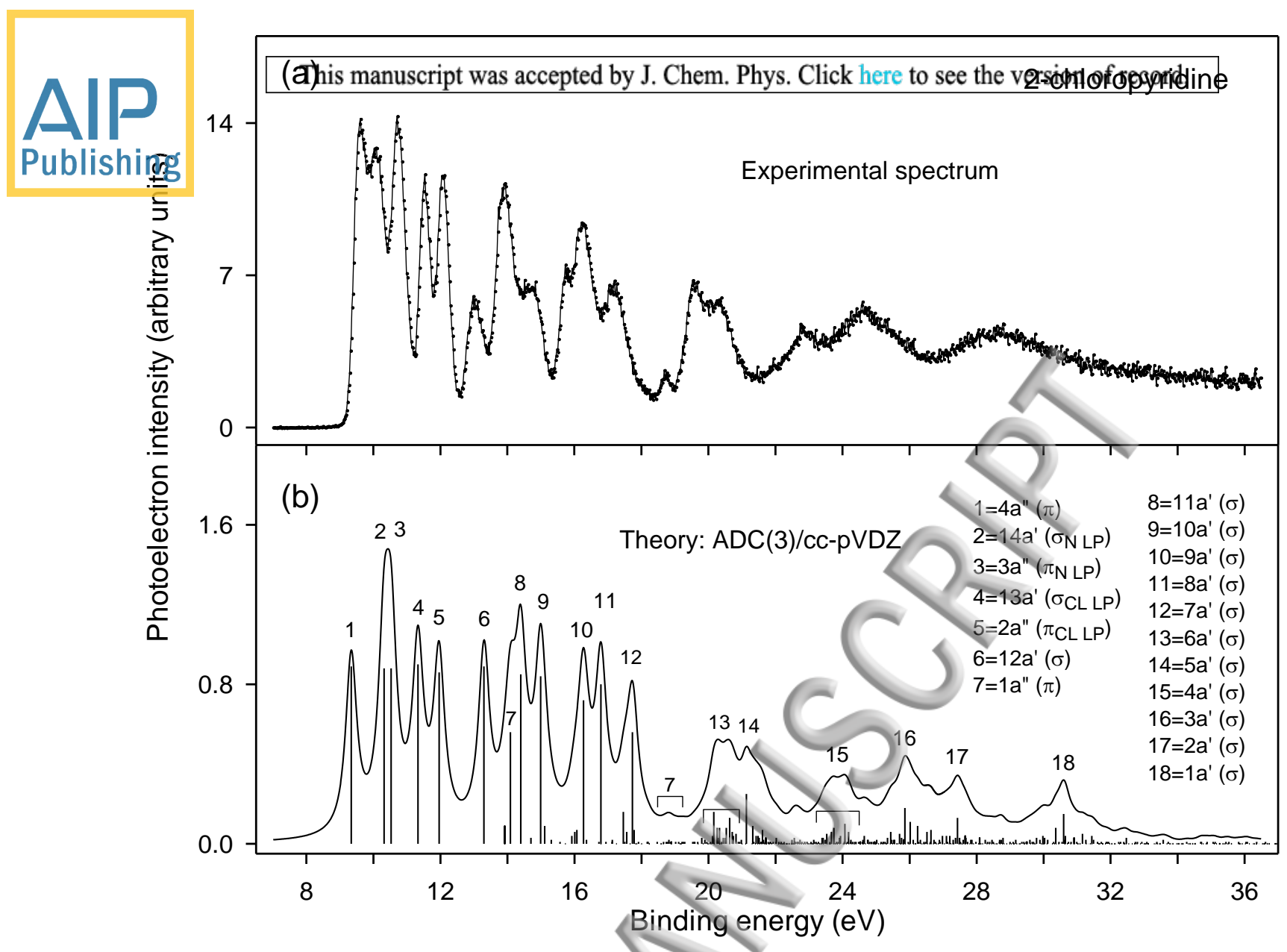




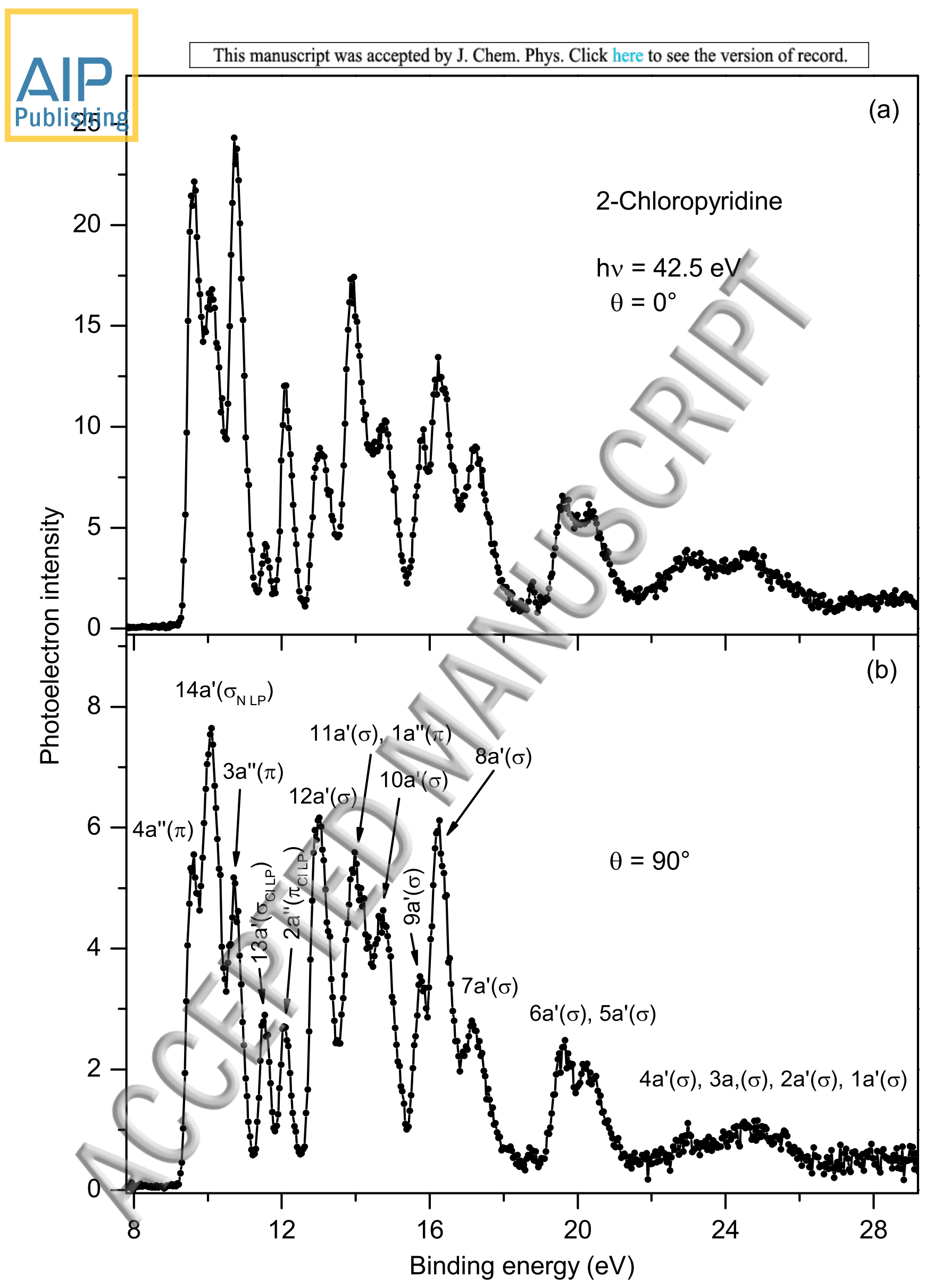




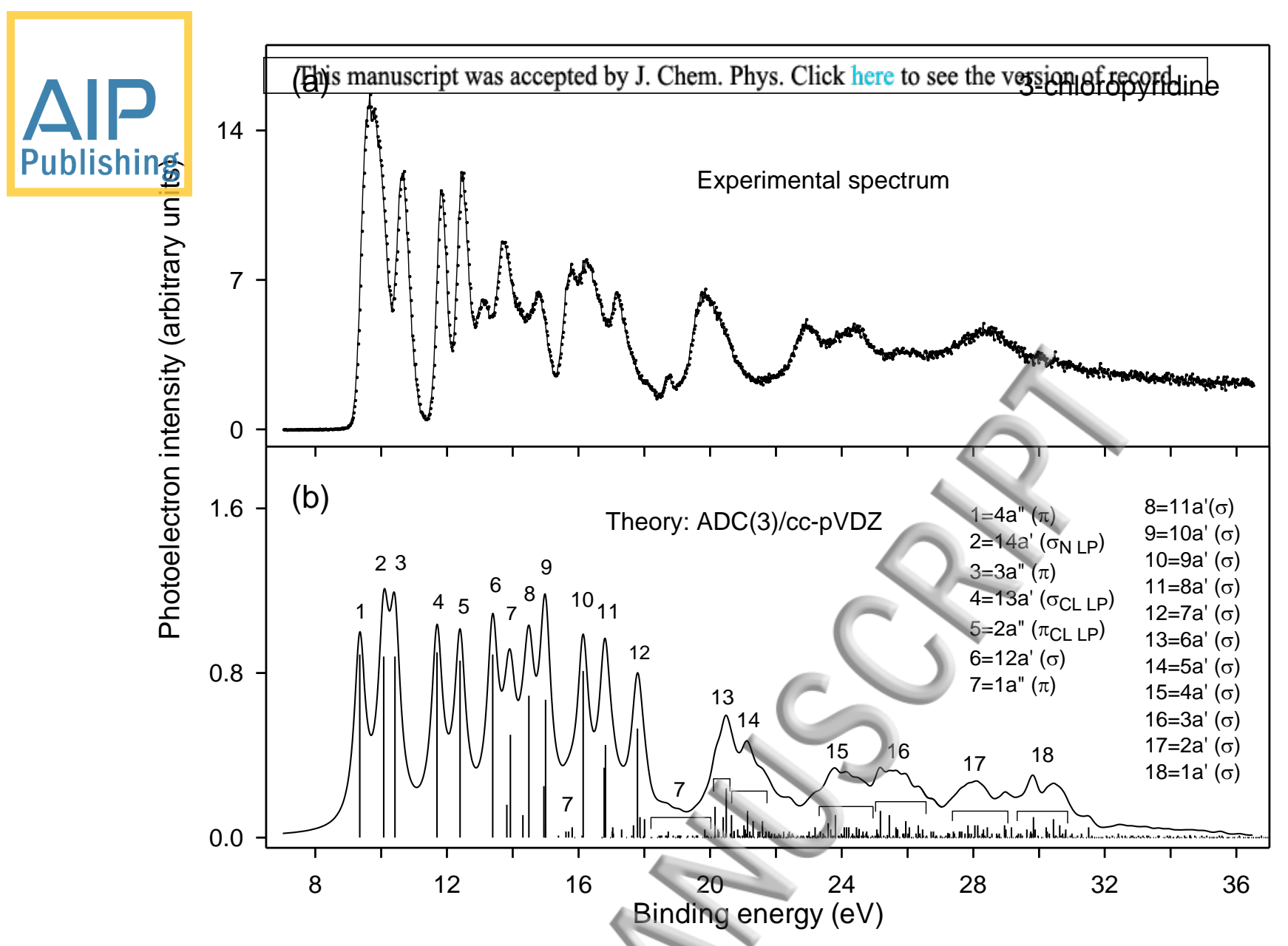




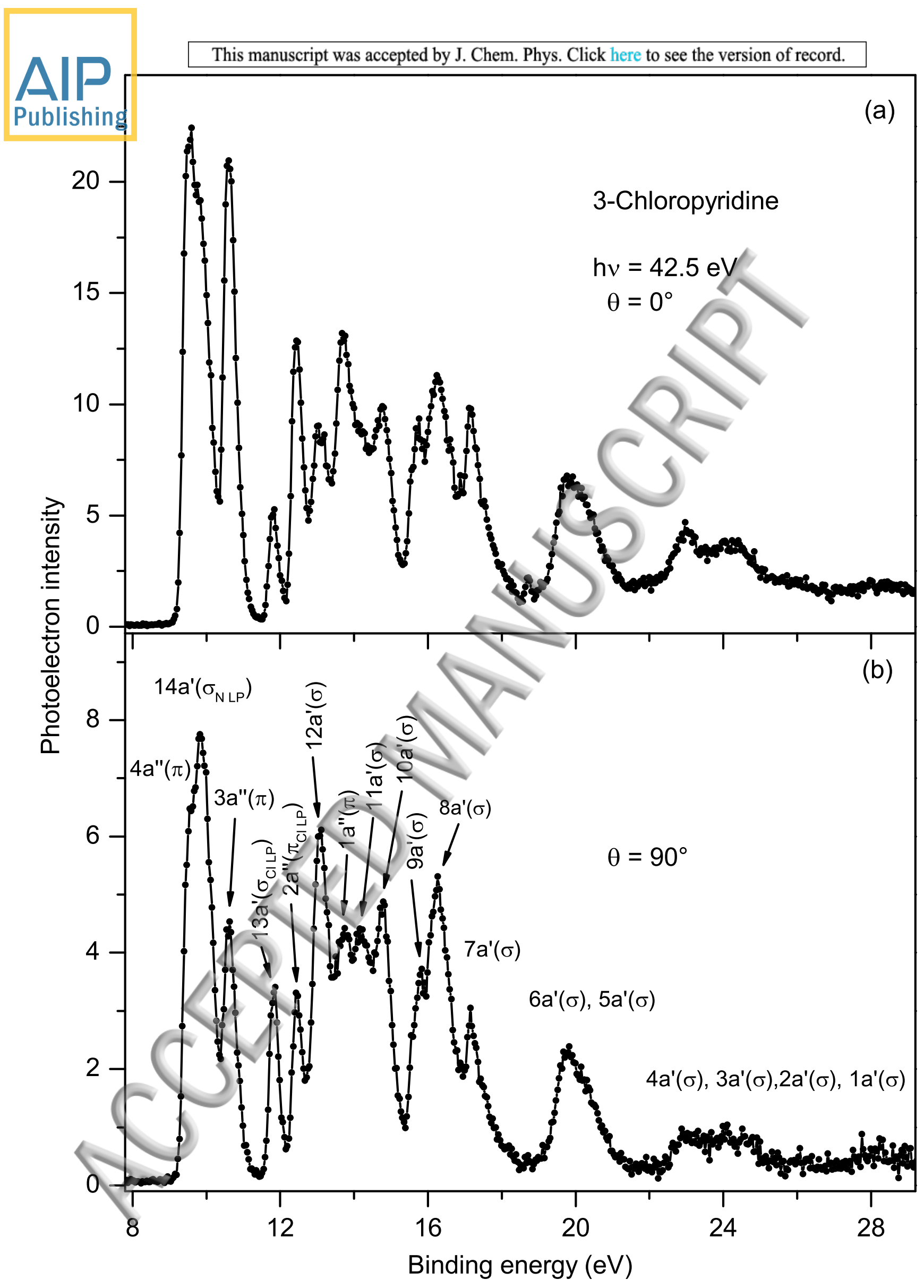




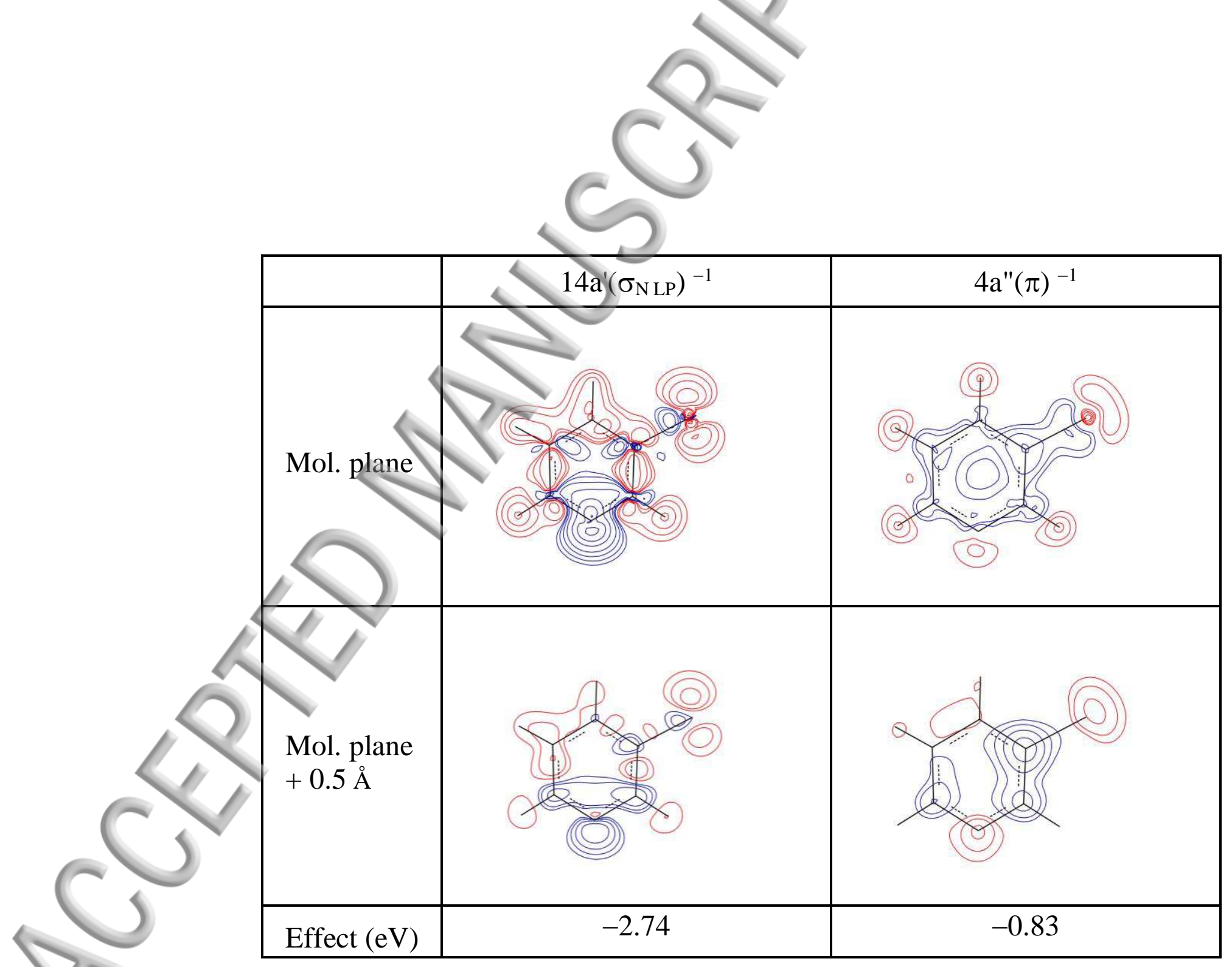




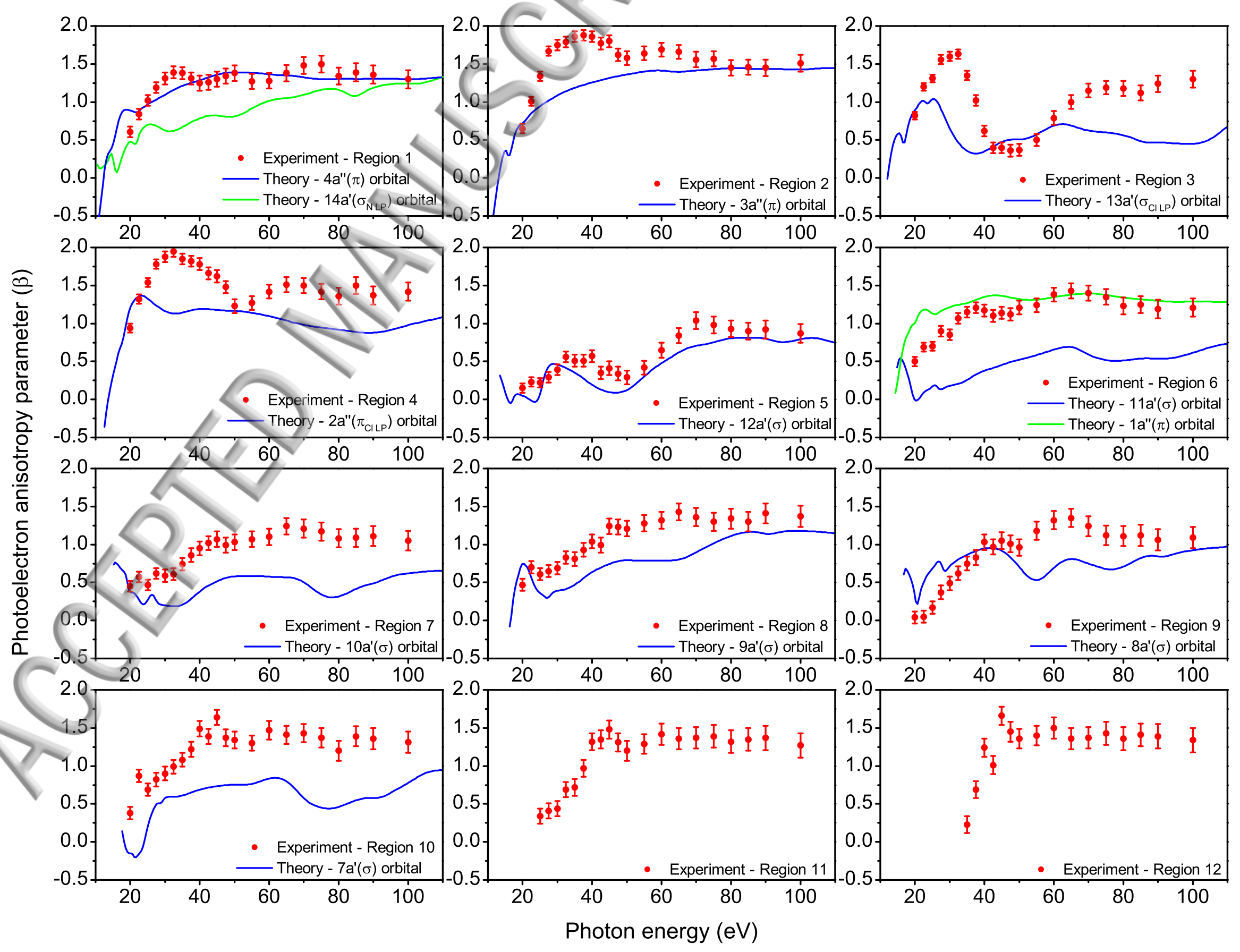



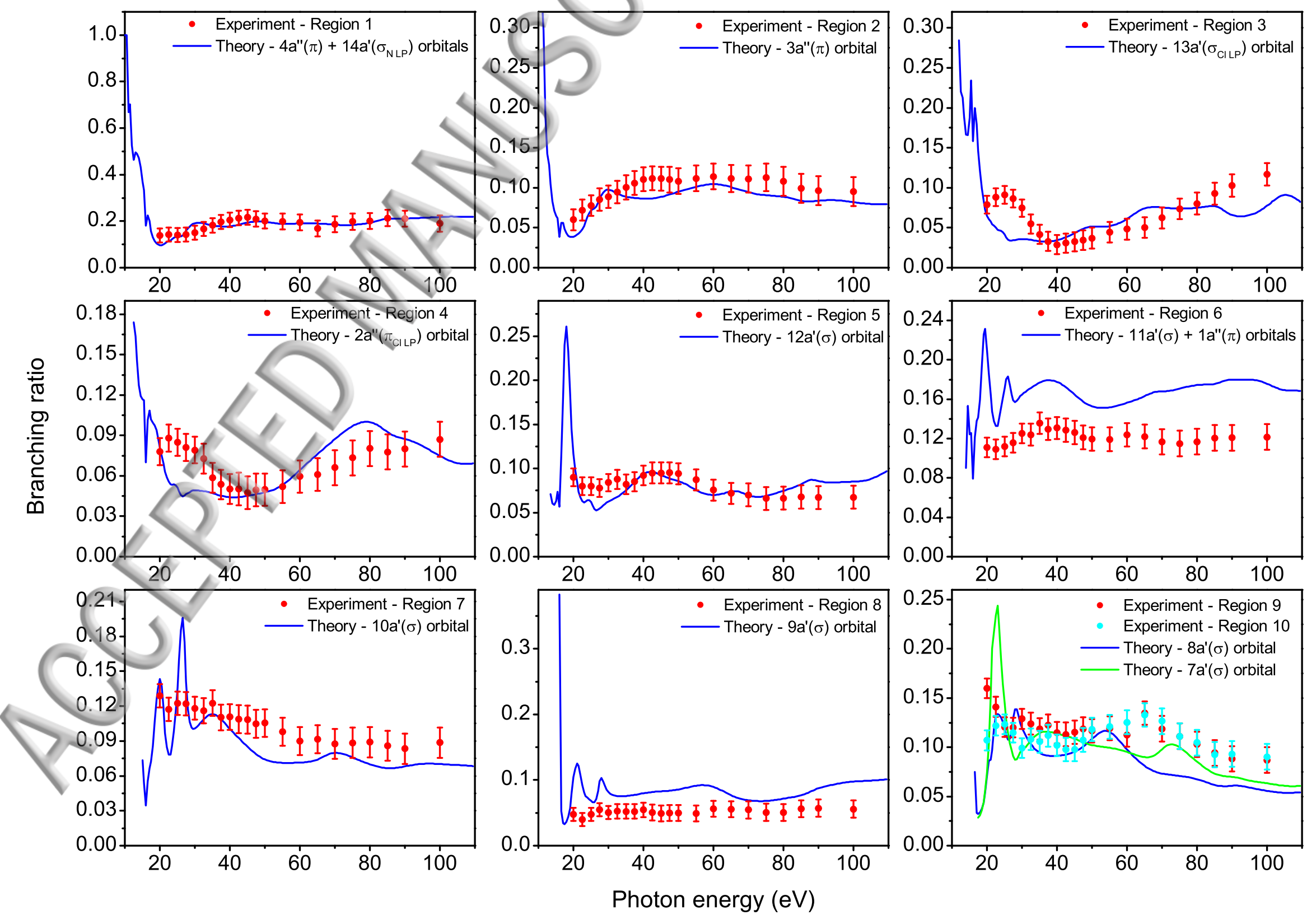